\title{
Construction of the energy matrix for complex atoms
}

\section{Part VII: High-performance computing in the case of terbium atom}

\author{
Magdalena Elantkowska ${ }^{1, a}$, Andrzej Sikorski ${ }^{2}, J$ arosław Ruczkowski², and Jerzy Dembczyński ${ }^{2}$ \\ 1 Institute of Materials Research and Quantum Engineering, Faculty of Technical Physics, Poznan University of Technology, \\ Piotrowo 3, 60-965 Poznań, Poland \\ 2 Institute of Control and Information Engineering, Faculty of Electrical Engineering, Poznan University of Technology, Piotrowo \\ 3A, 60-965 Poznań, Poland
}

Received: 25 January 2017 / Revised: 14 February 2017

Published online: 22 March 2017

(c) The Author(s) 2017. This article is published with open access at Springerlink.com

\begin{abstract}
We report the fine structure analysis of the even configurations system of atomic terbium in the complete set of $4 \mathrm{f}^{N}$-core states. For the purpose of the huge matrix diagonalization, we propose the methods utilizing the personal computer clusters and, alternatively, the Microsoft Azure cloud computing. The implementation of these approaches is presented.
\end{abstract}

\section{Introduction}

The present paper is the seventh one in the series of our systematic investigations on the structure of complex atom. The six of previous works entitled Construction of the energy matrix for complex atoms can be characterized as follows:

- Part I: general remarks on our method of semi-empirical analysis of complex atoms [1].

- Part II: introduction of the explicit formulae for inter-configuration interactions between the configurations containing up to four open shells [2].

- Parts III and IV: discussion of effective electrostatic interactions caused by the excitation of two equivalent electrons, as well as of one electron from a closed shell into an open shell or an empty shell [3,4].

- Part V: discussion of electrostatically correlated spin-orbit interactions, as well as electrostatically correlated hyperfine interactions caused by the excitation of one electrons from a closed shell into an open shell or an empty shell [5].

- Part VI: discussion of contributions to the hyperfine structure originating from core polarization effects [6].

The aim of this paper is the application of our semi-empirical parametrization method to the analysis of a complex electronic system of the elements with partially filled $4 \mathrm{f}$ or $5 \mathrm{f}$ electron shells.

Our earlier experience in semi-empirical calculations for the above-mentioned elements was related to the americium, europium and praseodymium atoms [7-10]. On the basis of these studies, we observed that inclusion of higher configurations into the energy matrix and the extension of terms for the $4 \mathrm{f}^{N}$-core systematically improved the agreement between the calculated and experimental energy level values. A large amount of new experimental data of the hyperfine structure of the terbium atom, recently published by our experimental group [11-13], directs once again, our interest in the research of the rare-earth spectra. In ref. [13], recently published (2017), a parametric study of the fine structure (fs) and of the hyperfine structure (hfs) for even-parity configurations of atomic terbium (Tb I) was presented. We performed semi-empirical calculations on the basis of 7 even-parity configurations: $4 \mathrm{f}^{8} 5 \mathrm{~d}^{3}, 4 \mathrm{f}^{8} 5 \mathrm{~d}^{2} 6 \mathrm{~s}, 4 \mathrm{f}^{8} 5 \mathrm{~d} 6 \mathrm{~s}^{2}, 4 \mathrm{f}^{9} 6 \mathrm{~s} 6 \mathrm{p}, 4 \mathrm{f}^{9} 6 \mathrm{~s} 7 \mathrm{p}, 4 \mathrm{f}^{9} 6 \mathrm{~s} 8 \mathrm{p}$ and $4 \mathrm{f}^{9} 5 \mathrm{~d} 6 \mathrm{p}$. In that work, we restricted the $4 \mathrm{f}^{8}$-core to 13 terms

\footnotetext{
a e-mail: magdalena.elantkowska@put.poznan.pl
} 
$\left({ }^{7} \mathrm{~F}+{ }^{5} \mathrm{~S}+{ }^{5} \mathrm{P}+{ }^{5} \mathrm{D} 1,2,3+{ }^{5} \mathrm{~F} 1,2+{ }^{5} \mathrm{G} 1,2,3+{ }^{5} \mathrm{H} 1,2\right)$ and the $4 \mathrm{f}^{9}$-core to 3 terms $\left({ }^{6} \mathrm{P}+{ }^{6} \mathrm{~F}+{ }^{6} \mathrm{H}\right)$. The considered system included 707 states for $J$ quantum number ranging from $1 / 2$ to $21 / 2$.

In 2001, Zhang et al. reported the experimental and theoretical studies of Dy III [14]. In theoretical calculations, by means of the Cowan-code package [15,16], the adopted set of $4 \mathrm{f}^{10}, 4 \mathrm{f}^{9} 6 \mathrm{p}$ and $4 \mathrm{f}^{9} 5 \mathrm{~d}, 4 \mathrm{f}^{9} 6 \mathrm{~s}$ configurations represented a total of 3549 possible energy levels. The same approach was used for calculations in doubly ionized europium [17, 18]. The configurations considered in the calculations were $4 \mathrm{f}^{7}, 4 \mathrm{f}^{6} 6 \mathrm{p}$ for the odd parity and $4 \mathrm{f}^{6} 5 \mathrm{~d}, 4 \mathrm{f}^{6} 6 \mathrm{~s}$ for the even parity. This model contained a total number of 5323 possible energy levels.

In the current work we resigned from the previously mentioned limitations and performed the calculations for the terbium atom in the full number of $4 \mathrm{f}^{8}$ - and $4 \mathrm{f}^{9}$-core states. In this case, the system contained 74418 possible energy levels corresponding to $J$ values ranging from $1 / 2$ to $31 / 2$. The maximum rank of a single submatrix, for $J=9 / 2$, was 9936 . Such huge energy matrix for the rare-earth element was not previously considered.

It is clear that such a problem requires high-performance computing (HPC), concerning both CPU (Central Processing Unit) speed and memory allocation. The obvious choice would seem the supercomputing centres, but due to the batch queueing mode they are not optimal for iterative calculations requiring continuous interaction with the user. Therefore we propose alternative methods, allowing to use one's own computer clusters, generally available in the scientific laboratories. Another possibility is to use the Microsoft Azure cloud computing.

The essential aim of this work is therefore to answer the following questions:

- What impact on the results of the semi-empirical calculations has a consideration of the complete set of $\mathrm{nf}^{N}$-core states?

- Can such calculations be performed within an acceptable time without the use of high-power computers?

\section{Computational procedures}

The construction of the fine-structure energy matrix requires calculation of numerous integrals dependent on the angular coordinates and various radial integrals [1]. The angular-dependent coefficients can be exactly determined, which is not possible in the case of radial integrals. Therefore, the matrix elements of the Hamiltonian are considered as linear combinations of radial integrals $P(k)$, where the angular integrals $\alpha_{i j}(k)$ serve as the coefficients of expansions,

$$
H_{i j}=\sum_{k} \alpha_{i j}(k) P(k),
$$

where $i$ and $j$ denote all possible $S L$ states for the considered configuration system, and $k$ represents the summation over all parameters described the interactions of the first- and higher-order perturbation theory, predicted by quantum mechanics.

The matrix elements of all operators are diagonal in $J$ quantum number, therefore the energy matrix can be divided into submatrices corresponding to possible values of $J$. The rank of each submatrix is determined by the number of states with the same value of the total angular momentum $J$. For each submatrix a secular determinant is constructed:

$$
\left|\begin{array}{ccccc}
H_{11}-E_{J} & H_{12} & H_{13} & \ldots & H_{1 j} \\
H_{21} & H_{22}-E_{J} & \ldots & \ldots & H_{2 j} \\
H_{31} & H_{32} & H_{33}-E_{J} \ldots & H_{3 j} \\
\ldots & \ldots & \ldots & \ldots & \ldots \\
H_{i 1} & \ldots & \ldots & \ldots H_{i j}-E_{J}
\end{array}\right|=0 .
$$

The above-mentioned determinant, after expansion, is a polynomial of degree $i$ with unknown energy values $E_{J}$. Every root of the polynomial approximates one of the possible eigenvalues of the Hamiltonian. In an iterative fitting procedure of experimental and calculated energy values the radial parameters and the eigenfunctions amplitudes are determined.

The main problem is the optimization of diagonalization procedures in such a way that the computing time of a single iteration will be as short as possible. 


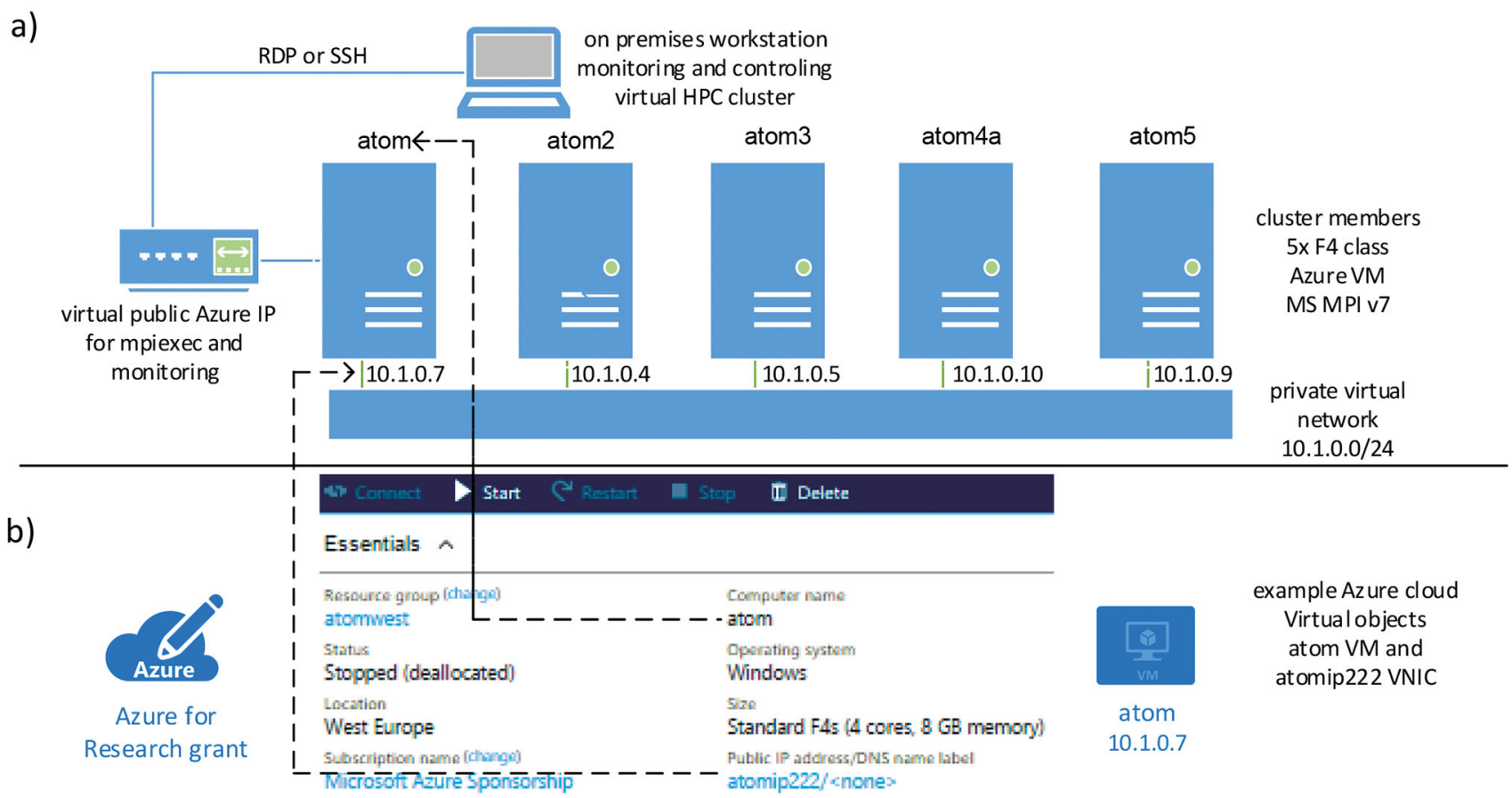

Fig. 1. HPC cluster for semi empirical terbium electronic structure calculations: (a) logical structure of Message Passing Interface (MPI) based cluster; (b) an example implementation with a set of Azure VMs. Please note that there are three possible deployment variants: Azure VMs, Azure Batch and local network with regular 4 core PCs. Designations used in the figure are only examples.

\subsection{HPC calculations on Azure cloud}

The underlying calculations were ported to Azure cloud, which resulted in a considerable performance boost. Indeed, the original calculations, implemented in a conventional sequential manner, required about ten hours per iteration, while it was reduced to less than three minutes when reprogrammed and launched on a cloud cluster. The achieved performance improvement was the result of individual $H$ matrix blocks multithreaded diagonalization and, to an even greater degree, distribution over multiple nodes. The individual components of the block diagonal matrix were processed locally on Azure Virtual Machines (VMs) and only a small amount of data had to be exchanged among the cluster members. For now the local parallel processing is delegated to the multithreaded version of Intel Math Kernel Library (MKL), however in our future work we are going to develop our own tridiagonalization routines since MKL SSYEV procedure scales poorly on nodes with more than 4 CPU cores.

There are three possible variants considered for the implementation of the cluster (fig. 1). The most evident candidate, namely the Azure Batch [19], proved not suitable for our purposes since it requires data transfers of angular coefficients every time the process is initiated. The Azure File Storage shares did not result in sufficient improvement providing rather disappointing input-output (IO) rates. In contrast, the other two options, Azure VMs and a local cluster, performed well, the latter even when based on network file share. In the code listed below, we give the launching routine for static Azure cluster (cf. fig. 1 for nodes' names). The nodes are started asynchronously, reducing the time overhead (which was prohibitive in the Azure Batch service). Please note that it is essential to efficiently allocate and deallocate the cluster as the user is charged for VM time, not for the actual computation. We are going to publish a simple, "one click" open-source software application supporting easy configuration and control of the Azure VM-based HPC clusters:

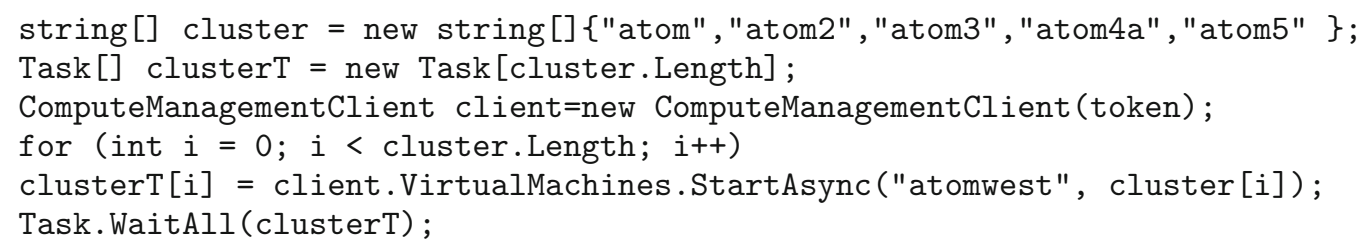




\subsection{Coordination of distributed tasks}

Our cloud-oriented approach is based on two fundamental ideas, that enable efficient distribution of the workload while incurring limited communication and synchronization overhead. First, let us observe the $H$ matrix is block diagonal (a very common case in quantum physics) allowing parallel processing of its components. Second, the semiempirical tuning of radial coefficient requires only relatively small amount of data in the input file, which we transfer to one designated node responsible for the data collection and calculation of new radial values. Evidently (see sect. 2), the $H$ matrix is a linear combination of radial and angular factors, thus the Jacobian involved in the radial calculation can be easily reconstructed at the mentioned "main" node. Simply put, member nodes transfer partial Jacobians and block until the main node returns new radial coefficients. Technically an MPI_Barrier is used for cluster coordination. This scheme can be considered an application of well-known multiway rendez-vous design pattern, whose two-way variant is present in ADA programming language. Disappointing performance of ssyev routine on nodes with 8 or more CPU cores made the efficient workload distribution a key performance factor in our project.

\section{Results}

The results of fs analysis are presented in table 1 . The first two columns list the values of the experimental and calculated level energies. The energy and $g_{J}$ values are taken from the NIST Atomic Spectra Database [20], whose primary source is the monograph by Martin et al. [21]. The fine-structure least-squares fit, using all 99 known experimental even-parity energy levels and 26 fitted parameters, resulted in a mean error for the energy level values of $\sigma(E)=37 \mathrm{~cm}^{-1}$. The following four columns of table 1 contain the strongest and second strongest fine-structure components, as well as the corresponding percentages. Experimental $g_{j}$-Landé factors are known for 66 energy levels. In most cases, the discrepancies are observed in the third or the second decimal position (27 and 36 values, respectively), confirming the good quality of the obtained wave functions. In columns 7 and 8 of table 1 the calculated $g_{J}$ values are compared with the experimental ones.

A comparison of current results with those published earlier [13] leads to the following conclusions. First, significantly better compliance of the calculated and the experimental energy values in the region up to $17000 \mathrm{~cm}^{-1}$ is noticeable. This is also confirmed by the much smaller root mean square value. Secondly, much more reliable description of the levels above $17000 \mathrm{~cm}^{-1}$ was achieved. In this energy range, our previous results [13], due to the applied limitations, indicated that the positions of the $4 \mathrm{f}^{9} 6 \mathrm{~s} 6 \mathrm{p}$ configuration states were not calculated correctly.

However, the final confirmation of the levels designations will be possible after the performing of the hyperfinestructure parametrization in the same, complete basis states. This requires the optimization and the adaptation of hyperfine-structure computational procedures for such a huge matrices. Encouraging results obtained in this work motivate us to undertake these tasks.

\section{Conclusions}

The preliminary results of fine-structure calculations indicate the need for considering the complete set of $4 \mathrm{f}^{N}$ core states. The comparison with the analysis, recently published [13], showed that the truncation of the number of $4 \mathrm{f}^{8}$ and $4 \mathrm{f}^{9}$ states affected even the energy levels in the region of several thousands $\mathrm{cm}^{-1}$. Therefore, in our opinion, a reanalysis of the structure of rare-earth elements, conducted without any truncations, is highly recommended.

The way to achieve this purpose is the development of novelty kind of computational procedures optimization. Indeed, the MPI-based distribution and multithreaded diagonalization resulted in a 200 times performance boost over the original version, which enabled efficient execution of multiple session required to obtain desired radial coefficient accuracy. In our future work, we are going to investigate chances of overcoming the current memory bandwidth woes (i.e. the disappointing SSYEV performance on multicore CPU). We are also going to publish a user-friendly configuration assistant, facilitating streamlined configuration of Azure VM based HPC cluster, an attractive offer for the research community.

This work was performed in the framework of Microsoft Azure for Research grant awarded to Andrzej Sikorski. This work was partially supported by the Research Projects of the Polish Ministry of Sciences and Higher Education: 06/65/DSPB/0516 (ME) and $04 / 45 / \mathrm{DSPB} / 0148$ (JR and JD). 
Table 1. Comparison of the experimental and calculated energy values $\left[\mathrm{cm}^{-1}\right]$ for $\mathrm{Tb} \mathrm{I}$.

\begin{tabular}{|c|c|c|c|c|c|c|c|c|c|}
\hline$E_{\exp }$ & $E_{\text {calc }}$ & $\Delta E$ & $\%$ & Main comp. & $\%$ & Sec. comp. & $g_{J \text { calc }}$ & $g_{J \exp }$ & $\Delta g_{J}$ \\
\hline \multicolumn{10}{|l|}{$J=1 / 2$} \\
\hline 4018.210 & 4101 & -83 & 87.4 & $4 \mathrm{f}^{8}\left({ }^{7} \mathrm{~F}\right) 5 \mathrm{~d} 6 \mathrm{~s}^{2}{ }^{8} \mathrm{G}$ & 2.9 & $4 \mathrm{f}^{8}\left({ }^{5} \mathrm{D}\right) 5 \mathrm{~d} 6 \mathrm{~s}^{2}{ }^{6} \mathrm{~F}$ & -1.157 & -1.191 & -0.034 \\
\hline \multirow[t]{8}{*}{6259.090} & 6175 & 84 & 86.4 & $4 \mathrm{f}^{8}\left({ }^{7} \mathrm{~F}\right) 5 \mathrm{~d} 6 \mathrm{~s}^{2}{ }^{8} \mathrm{~F}$ & 2.8 & $4 \mathrm{f}^{8}\left({ }^{5} \mathrm{D}\right) 5 \mathrm{~d} 6 \mathrm{~s}^{2}{ }^{6} \mathrm{D}$ & 3.808 & 3.840 & 0.032 \\
\hline & 11212 & & 81.1 & $4 \mathrm{f}^{8}\left({ }^{7} \mathrm{~F}\right) 5 \mathrm{~d}^{2} 6 \mathrm{~s}^{10} \mathrm{G}$ & 8.6 & $4 \mathrm{f}^{8}\left({ }^{7} \mathrm{~F}\right) 5 \mathrm{~d}^{2} 6 \mathrm{~s}{ }^{10} \mathrm{G}$ & 4.372 & & \\
\hline & 11243 & & 81.6 & $4 \mathrm{f}^{8}\left({ }^{7} \mathrm{~F}\right) 5 \mathrm{~d} 6 \mathrm{~s}^{2}{ }^{6} \mathrm{~F}$ & 3.5 & $4 \mathrm{f}^{8}\left({ }^{7} \mathrm{~F}\right) 5 \mathrm{~d}^{2} 6 \mathrm{~s}{ }^{10} \mathrm{G}$ & -0.370 & & \\
\hline & 13501 & & 81.1 & $4 \mathrm{f}^{8}\left({ }^{7} \mathrm{~F}\right) 5 \mathrm{~d} 6 \mathrm{~s}^{2}{ }^{6} \mathrm{D}$ & 2.7 & $4 f^{8}\left({ }^{7} F\right) 5 d^{2} 6 s{ }^{6} D$ & 3.242 & & \\
\hline & 14787 & & 78.9 & $4 \mathrm{f}^{8}\left({ }^{7} \mathrm{~F}\right) 5 \mathrm{~d}^{2} 6 \mathrm{~s}{ }^{10} \mathrm{H}$ & 7.3 & $4 \mathrm{f}^{8}\left({ }^{7} \mathrm{~F}\right) 5 \mathrm{~d}^{2} 6 \mathrm{~s}^{8} \mathrm{G}$ & -1.790 & & \\
\hline & 15007 & & 48.6 & $4 \mathrm{f}^{8}\left({ }^{7} \mathrm{~F}\right) 5 \mathrm{~d}^{2} 6 \mathrm{~s}^{8} \mathrm{G}$ & 18.5 & $4 \mathrm{f}^{8}\left({ }^{7} \mathrm{~F}\right) 5 \mathrm{~d}^{2} 6 \mathrm{~s}^{8} \mathrm{G}$ & -1.215 & & \\
\hline & 16931 & & 61.4 & $4 \mathrm{f}^{8}\left({ }^{7} \mathrm{~F}\right) 5 \mathrm{~d}^{2} 6 \mathrm{~s}^{8} \mathrm{~F}$ & 18.1 & $4 \mathrm{f}^{8}\left({ }^{7} \mathrm{~F}\right) 5 \mathrm{~d}^{2} 6 \mathrm{~s}^{8} \mathrm{~F}$ & 3.737 & & \\
\hline & 20024 & & 82.4 & $4 \mathrm{f}^{8}\left({ }^{7} \mathrm{~F}\right) 5 \mathrm{~d}^{2} 6 \mathrm{~s}{ }^{10} \mathrm{G}$ & 9.3 & $4 \mathrm{f}^{8}\left({ }^{7} \mathrm{~F}\right) 5 \mathrm{~d}^{2} 6 \mathrm{~s}{ }^{10} \mathrm{G}$ & 4.594 & & \\
\hline \multicolumn{10}{|l|}{$J=3 / 2$} \\
\hline 3705.820 & 3761 & -55 & 80.1 & $4 \mathrm{f}^{8}\left({ }^{7} \mathrm{~F}\right) 5 \mathrm{~d} 6 \mathrm{~s}^{2}{ }^{8} \mathrm{G}$ & 8.6 & $4 \mathrm{f}^{8}\left({ }^{7} \mathrm{~F}\right) 5 \mathrm{~d} 6 \mathrm{~s}^{2}{ }^{8} \mathrm{~F}$ & 1.045 & 1.022 & -0.023 \\
\hline 5483.980 & 5481 & 3 & 42.4 & $4 \mathrm{f}^{8}\left({ }^{7} \mathrm{~F}\right) 5 \mathrm{~d} 6 \mathrm{~s}^{2}{ }^{8} \mathrm{~F}$ & 40.1 & $4 \mathrm{f}^{8}\left({ }^{7} \mathrm{~F}\right) 5 \mathrm{~d} 6 \mathrm{~s}^{2}{ }^{8} \mathrm{D}$ & 2.247 & 2.320 & 0.073 \\
\hline 6849.720 & 6887 & -37 & 48.8 & $4 \mathrm{f}^{8}\left({ }^{7} \mathrm{~F}\right) 5 \mathrm{~d} 6 \mathrm{~s}^{2}{ }^{8} \mathrm{D}$ & 37.5 & $4 \mathrm{f}^{8}\left({ }^{7} \mathrm{~F}\right) 5 \mathrm{~d} 6 \mathrm{~s}^{2}{ }^{8} \mathrm{~F}$ & 2.385 & 2.335 & -0.050 \\
\hline \multirow[t]{2}{*}{8336.310} & 8350 & -14 & 83.5 & $4 \mathrm{f}^{8}\left({ }^{7} \mathrm{~F}\right) 5 \mathrm{~d} 6 \mathrm{~s}^{2}{ }^{8} \mathrm{H}$ & 4.4 & $4 \mathrm{f}^{8}\left({ }^{7} \mathrm{~F}\right) 5 \mathrm{~d} 6 \mathrm{~s}^{2}{ }^{6} \mathrm{G}$ & -0.345 & -0.360 & -0.015 \\
\hline & 10765 & & 76.3 & $4 \mathrm{f}^{8}\left({ }^{7} \mathrm{~F}\right) 5 \mathrm{~d} 6 \mathrm{~s}^{2}{ }^{6} \mathrm{~F}$ & 5.1 & $4 \mathrm{f}^{8}\left({ }^{7} \mathrm{~F}\right) 5 \mathrm{~d} 6 \mathrm{~s}^{2}{ }^{6} \mathrm{G}$ & 1.045 & & \\
\hline \multirow[t]{13}{*}{10920.180} & 10899 & 21 & 77.4 & $4 \mathrm{f}^{8}\left({ }^{7} \mathrm{~F}\right) 5 \mathrm{~d}^{2} 6 \mathrm{~s}{ }^{10} \mathrm{G}$ & 8.0 & $4 \mathrm{f}^{8}\left({ }^{7} \mathrm{~F}\right) 5 \mathrm{~d}^{2} 6 \mathrm{~s}{ }^{10} \mathrm{G}$ & 2.150 & 2.145 & -0.005 \\
\hline & 12044 & & 72.4 & $4 \mathrm{f}^{8}\left({ }^{7} \mathrm{~F}\right) 5 \mathrm{~d} 6 \mathrm{~s}^{2}{ }^{6} \mathrm{G}$ & 4.9 & $4 \mathrm{f}^{8}\left({ }^{7} \mathrm{~F}\right) 5 \mathrm{~d} 6 \mathrm{~s}^{2}{ }^{8} \mathrm{H}$ & 0.094 & & \\
\hline & 13049 & & 72.0 & $4 \mathrm{f}^{8}\left({ }^{7} \mathrm{~F}\right) 5 \mathrm{~d} 6 \mathrm{~s}^{2}{ }^{6} \mathrm{D}$ & 6.0 & $4 \mathrm{f}^{8}\left({ }^{7} \mathrm{~F}\right) 5 \mathrm{~d} 6 \mathrm{~s}^{2}{ }^{6} \mathrm{P}$ & 1.844 & & \\
\hline & 13411 & & 79.0 & $4 \mathrm{f}^{8}\left({ }^{7} \mathrm{~F}\right) 5 \mathrm{~d}^{2} 6 \mathrm{~s}{ }^{10} \mathrm{~F}$ & 7.9 & $4 \mathrm{f}^{8}\left({ }^{7} \mathrm{~F}\right) 5 \mathrm{~d}^{2} 6 \mathrm{~s}{ }^{10} \mathrm{~F}$ & 3.081 & & \\
\hline & 14555 & & 32.6 & $4 \mathrm{f}^{8}\left({ }^{7} \mathrm{~F}\right) 5 \mathrm{~d}^{2} 6 \mathrm{~s}^{8} \mathrm{G}$ & 23.8 & $4 \mathrm{f}^{8}\left({ }^{7} \mathrm{~F}\right) 5 \mathrm{~d}^{2} 6 \mathrm{~s}{ }^{10} \mathrm{H}$ & 0.937 & & \\
\hline & 14702 & & 55.1 & $4 \mathrm{f}^{8}\left({ }^{7} \mathrm{~F}\right) 5 \mathrm{~d}^{2} 6 \mathrm{~s}{ }^{10} \mathrm{H}$ & 16.6 & $4 \mathrm{f}^{8}\left({ }^{7} \mathrm{~F}\right) 5 \mathrm{~d}^{2} 6 \mathrm{~s}{ }^{8} \mathrm{G}$ & 0.764 & & \\
\hline & 15388 & & 78.9 & $4 \mathrm{f}^{8}\left({ }^{7} \mathrm{~F}\right) 5 \mathrm{~d} 6 \mathrm{~s}^{2}{ }^{6} \mathrm{P}$ & 5.8 & $4 \mathrm{f}^{8}\left({ }^{7} \mathrm{~F}\right) 5 \mathrm{~d} 6 \mathrm{~s}^{2}{ }^{6} \mathrm{D}$ & 2.332 & & \\
\hline & 15679 & & 80.7 & $4 \mathrm{f}^{8}\left({ }^{7} \mathrm{~F}\right) 5 \mathrm{~d}^{2} 6 \mathrm{~s}{ }^{10} \mathrm{I}$ & 11.1 & $4 \mathrm{f}^{8}\left({ }^{7} \mathrm{~F}\right) 5 \mathrm{~d}^{2} 6 \mathrm{~s}{ }^{10} \mathrm{H}$ & -0.577 & & \\
\hline & 16704 & & 48.5 & $4 \mathrm{f}^{8}\left({ }^{7} \mathrm{~F}\right) 5 \mathrm{~d}^{2} 6 \mathrm{~s}^{8} \mathrm{~F}$ & 14.1 & $4 \mathrm{f}^{8}\left({ }^{7} \mathrm{~F}\right) 5 \mathrm{~d}^{2} 6 \mathrm{~s}^{8} \mathrm{~F}$ & 1.913 & & \\
\hline & 17936 & & 73.9 & $4 \mathrm{f}^{8}\left({ }^{7} \mathrm{~F}\right) 5 \mathrm{~d}^{2} 6 \mathrm{~s}^{8} \mathrm{H}$ & 13.1 & $4 \mathrm{f}^{8}\left({ }^{7} \mathrm{~F}\right) 5 \mathrm{~d}^{2} 6 \mathrm{~s}^{8} \mathrm{H}$ & -0.316 & & \\
\hline & 19285 & & 64.3 & $4 f^{8}\left({ }^{7} F\right) 5 d^{2} 6 s^{8} D$ & 11.2 & $4 f^{8}\left({ }^{7} F\right) 5 d^{2} 6 s^{8} D$ & 2.662 & & \\
\hline & 19840 & & 81.1 & $4 \mathrm{f}^{8}\left({ }^{7} \mathrm{~F}\right) 5 \mathrm{~d}^{2} 6 \mathrm{~s}{ }^{10} \mathrm{G}$ & 9.3 & $4 \mathrm{f}^{8}\left({ }^{7} \mathrm{~F}\right) 5 \mathrm{~d}^{2} 6 \mathrm{~s}{ }^{10} \mathrm{G}$ & 2.111 & & \\
\hline & 20480 & & 32.2 & $4 \mathrm{f}^{8}\left({ }^{7} \mathrm{~F}\right) 5 \mathrm{~d}^{2} 6 \mathrm{~s}{ }^{6} \mathrm{~F}$ & 16.4 & $4 \mathrm{f}^{8}\left({ }^{7} \mathrm{~F}\right) 5 \mathrm{~d}^{2} 6 \mathrm{~s}{ }^{6} \mathrm{D}$ & 1.137 & & \\
\hline \multicolumn{10}{|l|}{$J=5 / 2$} \\
\hline 3174.575 & 3190 & -15 & 67.4 & $4 \mathrm{f}^{8}\left({ }^{7} \mathrm{~F}\right) 5 \mathrm{~d} 6 \mathrm{~s}^{2}{ }^{8} \mathrm{G}$ & 17.8 & $4 \mathrm{f}^{8}\left({ }^{7} \mathrm{~F}\right) 5 \mathrm{~d} 6 \mathrm{~s}^{2}{ }^{8} \mathrm{~F}$ & 1.374 & 1.355 & -0.019 \\
\hline 4695.505 & 4708 & -13 & 48.6 & $4 \mathrm{f}^{8}\left({ }^{7} \mathrm{~F}\right) 5 \mathrm{~d} 6 \mathrm{~s}^{2}{ }^{8} \mathrm{D}$ & 22.7 & $4 \mathrm{f}^{8}\left({ }^{7} \mathrm{~F}\right) 5 \mathrm{~d} 6 \mathrm{~s}^{2}{ }^{8} \mathrm{~F}$ & 1.803 & 1.831 & 0.028 \\
\hline 6801.190 & 6807 & -6 & 47.3 & $4 \mathrm{f}^{8}\left({ }^{7} \mathrm{~F}\right) 5 \mathrm{~d} 6 \mathrm{~s}^{2}{ }^{8} \mathrm{~F}$ & 33.6 & $4 \mathrm{f}^{8}\left({ }^{7} \mathrm{~F}\right) 5 \mathrm{~d} 6 \mathrm{~s}^{2}{ }^{8} \mathrm{D}$ & 1.811 & 1.800 & -0.011 \\
\hline 8130.680 & 8131 & -1 & 80.9 & $4 \mathrm{f}^{8}\left({ }^{7} \mathrm{~F}\right) 5 \mathrm{~d} 6 \mathrm{~s}^{2}{ }^{8} \mathrm{H}$ & 5.1 & $4 \mathrm{f}^{8}\left({ }^{7} \mathrm{~F}\right) 5 \mathrm{~d} 6 \mathrm{~s}^{2}{ }^{6} \mathrm{G}$ & 0.714 & 0.705 & -0.009 \\
\hline 10030.350 & 10037 & -6 & 70.4 & $4 \mathrm{f}^{8}\left({ }^{7} \mathrm{~F}\right) 5 \mathrm{~d} 6 \mathrm{~s}^{2}{ }^{6} \mathrm{~F}$ & 7.9 & $4 \mathrm{f}^{8}\left({ }^{7} \mathrm{~F}\right) 5 \mathrm{~d} 6 \mathrm{~s}^{2}{ }^{6} \mathrm{G}$ & 1.297 & 1.305 & 0.008 \\
\hline \multirow[t]{3}{*}{10456.670} & 10424 & 32 & 69.8 & $4 \mathrm{f}^{8}\left({ }^{7} \mathrm{~F}\right) 5 \mathrm{~d}^{2} 6 \mathrm{~s}{ }^{10} \mathrm{G}$ & 11.6 & $4 \mathrm{f}^{8}\left({ }^{7} \mathrm{~F}\right) 5 \mathrm{~d}^{2} 6 \mathrm{~s}{ }^{10} \mathrm{~F}$ & 1.803 & 1.800 & -0.003 \\
\hline & 11577 & & 63.1 & $4 \mathrm{f}^{8}\left({ }^{7} \mathrm{~F}\right) 5 \mathrm{~d} 6 \mathrm{~s}^{2}{ }^{6} \mathrm{G}$ & 7.7 & $4 \mathrm{f}^{8}\left({ }^{7} \mathrm{~F}\right) 5 \mathrm{~d} 6 \mathrm{~s}^{2}{ }^{6} \mathrm{D}$ & 0.953 & & \\
\hline & 11892 & & 81.8 & $4 \mathrm{f}^{8}\left({ }^{7} \mathrm{~F}\right) 5 \mathrm{~d} 6 \mathrm{~s}^{2}{ }^{8} \mathrm{P}$ & 3.5 & $4 \mathrm{f}^{8}\left({ }^{7} \mathrm{~F}\right) 5 \mathrm{~d} 6 \mathrm{~s}^{2}{ }^{8} \mathrm{D}$ & 2.244 & & \\
\hline \multirow[t]{7}{*}{12296.45} & 12262 & 35 & 52.1 & $4 \mathrm{f}^{8}\left({ }^{7} \mathrm{~F}\right) 5 \mathrm{~d} 6 \mathrm{~s}^{2}{ }^{6} \mathrm{D}$ & 16.0 & $4 \mathrm{f}^{8}\left({ }^{7} \mathrm{~F}\right) 5 \mathrm{~d} 6 \mathrm{~s}^{2}{ }^{6} \mathrm{P}$ & 1.605 & & \\
\hline & 13076 & & 62.1 & $4 \mathrm{f}^{8}\left({ }^{7} \mathrm{~F}\right) 5 \mathrm{~d}^{2} 6 \mathrm{~s}{ }^{10} \mathrm{~F}$ & 10.1 & $4 \mathrm{f}^{8}\left({ }^{7} \mathrm{~F}\right) 5 \mathrm{~d}^{2} 6 \mathrm{~s}{ }^{10} \mathrm{D}$ & 2.153 & & \\
\hline & 13211 & & 82.0 & $4 \mathrm{f}^{8}\left({ }^{7} \mathrm{~F}\right) 5 \mathrm{~d} 6 \mathrm{~s}^{2}{ }^{6} \mathrm{H}$ & 3.6 & $4 \mathrm{f}^{8}\left({ }^{5} \mathrm{D}\right) 5 \mathrm{~d} 6 \mathrm{~s}^{2}{ }^{4} \mathrm{G}$ & 0.330 & & \\
\hline & 14029 & & 39.5 & $4 \mathrm{f}^{8}\left({ }^{7} \mathrm{~F}\right) 5 \mathrm{~d}^{2} 6 \mathrm{~s}^{8} \mathrm{G}$ & 18.3 & $4 \mathrm{f}^{8}\left({ }^{7} \mathrm{~F}\right) 5 \mathrm{~d}^{2} 6 \mathrm{~s}{ }^{8} \mathrm{G}$ & 1.352 & & \\
\hline & 14356 & & 65.4 & $4 \mathrm{f}^{8}\left({ }^{7} \mathrm{~F}\right) 5 \mathrm{~d} 6 \mathrm{~s}^{2}{ }^{6} \mathrm{P}$ & 15.6 & $4 \mathrm{f}^{8}\left({ }^{7} \mathrm{~F}\right) 5 \mathrm{~d} 6 \mathrm{~s}^{2}{ }^{6} \mathrm{D}$ & 1.826 & & \\
\hline & 14437 & & 63.9 & $4 \mathrm{f}^{8}\left({ }^{7} \mathrm{~F}\right) 5 \mathrm{~d}^{2} 6 \mathrm{~s}{ }^{10} \mathrm{H}$ & 20.1 & $4 \mathrm{f}^{8}\left({ }^{7} \mathrm{~F}\right) 5 \mathrm{~d}^{2} 6 \mathrm{~s}{ }^{10} \mathrm{I}$ & 1.096 & & \\
\hline & 15683 & & 70.1 & $4 \mathrm{f}^{8}\left({ }^{7} \mathrm{~F}\right) 5 \mathrm{~d}^{2} 6 \mathrm{~s}{ }^{10} \mathrm{I}$ & 19.8 & $4 \mathrm{f}^{8}\left({ }^{7} \mathrm{~F}\right) 5 \mathrm{~d}^{2} 6 \mathrm{~s}{ }^{10} \mathrm{H}$ & 0.679 & & \\
\hline
\end{tabular}


Table 1. Continued.

\begin{tabular}{|c|c|c|c|c|c|c|c|c|c|}
\hline$E_{\exp }$ & $E_{\text {calc }}$ & $\Delta E$ & $\%$ & Main comp. & $\%$ & Sec. comp. & $g_{J \text { calc }}$ & $g_{J \exp }$ & $\Delta g_{J}$ \\
\hline & 16000 & & 23.3 & $4 \mathrm{f}^{8}\left({ }^{7} \mathrm{~F}\right) 5 \mathrm{~d}^{2} 6 \mathrm{~s}{ }^{10} \mathrm{D}$ & 15.2 & $4 \mathrm{f}^{8}\left({ }^{7} \mathrm{~F}\right) 5 \mathrm{~d}^{2} 6 \mathrm{~s}^{8} \mathrm{D}$ & 2.047 & & \\
\hline & 16148 & & 54.7 & $4 \mathrm{f}^{8}\left({ }^{7} \mathrm{~F}\right) 5 \mathrm{~d}^{2} 6 \mathrm{~s}{ }^{10} \mathrm{D}$ & 11.3 & $4 f^{8}\left({ }^{7} F\right) 5 d^{2} 6 s^{8} F$ & 2.224 & & \\
\hline & 17449 & & 27.8 & $4 \mathrm{f}^{8}\left({ }^{7} \mathrm{~F}\right) 5 \mathrm{~d}^{2} 6 \mathrm{~s}^{8} \mathrm{P}$ & 21.3 & $4 \mathrm{f}^{8}\left({ }^{7} \mathrm{~F}\right) 5 \mathrm{~d}^{2} 6 \mathrm{~s}^{8} \mathrm{H}$ & 1.591 & & \\
\hline & 17736 & & 35.8 & $4 \mathrm{f}^{8}\left({ }^{7} \mathrm{~F}\right) 5 \mathrm{~d}^{2} 6 \mathrm{~s}^{8} \mathrm{H}$ & 10.6 & $4 \mathrm{f}^{8}\left({ }^{7} \mathrm{~F}\right) 5 \mathrm{~d}^{2} 6 \mathrm{~s}^{8} \mathrm{P}$ & 1.053 & & \\
\hline & 18135 & & 87.8 & $4 \mathrm{f}^{8}\left({ }^{7} \mathrm{~F}\right) 5 \mathrm{~d}^{2} 6 \mathrm{~s}{ }^{10} \mathrm{D}$ & 2.7 & $4 \mathrm{f}^{8}\left({ }^{5} \mathrm{D}\right) 5 \mathrm{~d}^{2} 6 \mathrm{~s}^{8} \mathrm{P}$ & 2.537 & & \\
\hline & 18749 & & 20.0 & $4 \mathrm{f}^{8}\left({ }^{5} \mathrm{D}\right) 5 \mathrm{~d} 6 \mathrm{~s}^{2}{ }^{6} \mathrm{P}$ & 15.5 & $4 \mathrm{f}^{8}\left({ }^{5} \mathrm{D}\right) 5 \mathrm{~d} 6 \mathrm{~s}^{2}{ }^{6} \mathrm{P}$ & 1.763 & & \\
\hline & 18782 & & 60.3 & $4 f^{8}\left({ }^{7} F\right) 5 d^{2} 6 s^{8} I$ & 12.1 & $4 \mathrm{f}^{8}\left({ }^{7} \mathrm{~F}\right) 5 \mathrm{~d}^{2} 6 \mathrm{~s}^{8} \mathrm{H}$ & 0.158 & & \\
\hline & 19515 & & 22.6 & $4 \mathrm{f}^{8}\left({ }^{7} \mathrm{~F}\right) 5 \mathrm{~d}^{2} 6 \mathrm{~s}{ }^{6} \mathrm{~F}$ & 18.0 & $4 \mathrm{f}^{8}\left({ }^{7} \mathrm{~F}\right) 5 \mathrm{~d}^{2} 6 \mathrm{~s}{ }^{6} \mathrm{D}$ & 1.464 & & \\
\hline & 19546 & & 70.2 & $4 f^{8}\left({ }^{7} F\right) 5 d^{2} 6 s{ }^{10} G$ & 8.4 & $4 \mathrm{f}^{8}\left({ }^{7} \mathrm{~F}\right) 5 \mathrm{~d}^{2} 6 \mathrm{~s}{ }^{10} \mathrm{G}$ & 1.724 & & \\
\hline & 20166 & & 37.1 & $4 \mathrm{f}^{8}\left({ }^{7} \mathrm{~F}\right) 5 \mathrm{~d}^{2} 6 \mathrm{~s}^{8} \mathrm{D}$ & 15.7 & $4 \mathrm{f}^{8}\left({ }^{7} \mathrm{~F}\right) 5 \mathrm{~d}^{2} 6 \mathrm{~s}^{8} \mathrm{P}$ & 2.044 & & \\
\hline & 20479 & & 29.1 & $4 f^{8}\left({ }^{7} F\right) 5 d^{2} 6 s{ }^{6} G$ & 17.3 & $4 \mathrm{f}^{8}\left({ }^{7} \mathrm{~F}\right) 5 \mathrm{~d}^{2} 6 \mathrm{~s}{ }^{6} \mathrm{P}$ & 1.263 & & \\
\hline \multicolumn{10}{|l|}{$J=7 / 2$} \\
\hline 2419.480 & 2400 & 20 & 51.0 & $4 \mathrm{f}^{8}\left({ }^{7} \mathrm{~F}\right) 5 \mathrm{~d} 6 \mathrm{~s}^{2}{ }^{8} \mathrm{G}$ & 26.1 & $4 \mathrm{f}^{8}\left({ }^{7} \mathrm{~F}\right) 5 \mathrm{~d} 6 \mathrm{~s}^{2}{ }^{8} \mathrm{~F}$ & 1.488 & 1.477 & -0.012 \\
\hline 3819.850 & 3839 & -20 & 47.4 & $4 f^{8}\left({ }^{7} F\right) 5 d 6 s^{2}{ }^{8} D$ & 28.7 & $4 f^{8}\left({ }^{7} F\right) 5 d 6 s^{2}{ }^{8} G$ & 1.630 & 1.642 & 0.012 \\
\hline 6488.280 & 6464 & 24 & 51.7 & $4 \mathrm{f}^{8}\left({ }^{7} \mathrm{~F}\right) 5 \mathrm{~d} 6 \mathrm{~s}^{2}{ }^{8} \mathrm{~F}$ & 21.6 & $4 f^{8}\left({ }^{7} F\right) 5 d 6 s^{2}{ }^{8} D$ & 1.640 & 1.635 & -0.005 \\
\hline 7839.850 & 7823 & 17 & 76.6 & $4 f^{8}\left({ }^{7} F\right) 5 d 6 s^{2}{ }^{8} \mathrm{H}$ & 6.2 & $4 \mathrm{f}^{8}\left({ }^{7} \mathrm{~F}\right) 5 \mathrm{~d} 6 \mathrm{~s}^{2}{ }^{6} \mathrm{G}$ & 1.070 & 1.050 & -0.020 \\
\hline 8994.660 & 9015 & -21 & 62.0 & $4 \mathrm{f}^{8}\left({ }^{7} \mathrm{~F}\right) 5 \mathrm{~d} 6 \mathrm{~s}^{2}{ }^{6} \mathrm{~F}$ & 12.8 & $4 \mathrm{f}^{8}\left({ }^{7} \mathrm{~F}\right) 5 \mathrm{~d} 6 \mathrm{~s}^{2}{ }^{6} \mathrm{D}$ & 1.404 & 1.414 & 0.010 \\
\hline 9867.650 & 9830 & 38 & 61.5 & $4 \mathrm{f}^{8}\left({ }^{7} \mathrm{~F}\right) 5 \mathrm{~d}^{2} 6 \mathrm{~s}^{10} \mathrm{G}$ & 16.8 & $4 \mathrm{f}^{8}\left({ }^{7} \mathrm{~F}\right) 5 \mathrm{~d}^{2} 6 \mathrm{~s}{ }^{10} \mathrm{~F}$ & 1.684 & 1.680 & -0.004 \\
\hline \multirow[t]{2}{*}{10324.740} & 10314 & 10 & 74.0 & $4 \mathrm{f}^{8}\left({ }^{7} \mathrm{~F}\right) 5 \mathrm{~d} 6 \mathrm{~s}^{2}{ }^{8} \mathrm{P}$ & 8.4 & $4 \mathrm{f}^{8}\left({ }^{7} \mathrm{~F}\right) 5 \mathrm{~d} 6 \mathrm{~s}^{2}{ }^{8} \mathrm{D}$ & 1.894 & 1.916 & 0.022 \\
\hline & 10558 & & 27.4 & $4 \mathrm{f}^{8}\left({ }^{7} \mathrm{~F}\right) 5 \mathrm{~d} 6 \mathrm{~s}^{2}{ }^{6} \mathrm{D}$ & 26.0 & $4 \mathrm{f}^{8}\left({ }^{7} \mathrm{~F}\right) 5 \mathrm{~d} 6 \mathrm{~s}^{2}{ }^{6} \mathrm{P}$ & 1.470 & & \\
\hline 11107.07 & 11120 & -13 & 39.7 & $4 \mathrm{f}^{8}\left({ }^{7} \mathrm{~F}\right) 5 \mathrm{~d} 6 \mathrm{~s}^{2}{ }^{6} \mathrm{G}$ & 17.4 & $4 \mathrm{f}^{8}\left({ }^{7} \mathrm{~F}\right) 5 \mathrm{~d} 6 \mathrm{~s}^{2}{ }^{6} \mathrm{~F}$ & 1.298 & & \\
\hline 12250.99 & 12290 & -39 & 26.6 & $4 \mathrm{f}^{8}\left({ }^{7} \mathrm{~F}\right) 5 \mathrm{~d}^{2} 6 \mathrm{~s}{ }^{10} \mathrm{~F}$ & 22.5 & $4 \mathrm{f}^{8}\left({ }^{7} \mathrm{~F}\right) 5 \mathrm{~d}^{2} 6 \mathrm{~s}{ }^{10} \mathrm{D}$ & 1.936 & & \\
\hline 12645.32 & 12658 & -13 & 72.6 & $4 f^{8}\left({ }^{7} \mathrm{~F}\right) 5 \mathrm{~d} 6 \mathrm{~s}^{2}{ }^{6} \mathrm{H}$ & 3.6 & $4 \mathrm{f}^{8}\left({ }^{7} \mathrm{~F}\right) 5 \mathrm{~d} 6 \mathrm{~s}^{2}{ }^{8} \mathrm{H}$ & 0.919 & & \\
\hline 12714.050 & 12784 & -70 & 35.2 & $4 f^{8}\left({ }^{7} F\right) 5 d 6 s^{2}{ }^{6} D$ & 33.2 & $4 \mathrm{f}^{8}\left({ }^{7} \mathrm{~F}\right) 5 \mathrm{~d} 6 \mathrm{~s}^{2}{ }^{6} \mathrm{P}$ & 1.590 & & \\
\hline 13277.23 & 13288 & -10 & 27.2 & $4 f^{8}\left({ }^{7} F\right) 5 d^{2} 6 s^{8} G$ & 12.9 & $4 \mathrm{f}^{8}\left({ }^{7} \mathrm{~F}\right) 5 \mathrm{~d}^{2} 6 \mathrm{~s}^{8} \mathrm{G}$ & 1.528 & & \\
\hline \multirow[t]{17}{*}{13729.12} & 13732 & -3 & 31.6 & $4 \mathrm{f}^{8}\left({ }^{7} \mathrm{~F}\right) 5 \mathrm{~d}^{2} 6 \mathrm{~s}{ }^{10} \mathrm{P}$ & 19.4 & $4 \mathrm{f}^{8}\left({ }^{7} \mathrm{~F}\right) 5 \mathrm{~d}^{2} 6 \mathrm{~s}{ }^{10} \mathrm{~F}$ & 1.861 & & \\
\hline & 14150 & & 36.8 & $4 \mathrm{f}^{8}\left({ }^{7} \mathrm{~F}\right) 5 \mathrm{~d}^{2} 6 \mathrm{~s}{ }^{10} \mathrm{H}$ & 18.5 & $4 \mathrm{f}^{8}\left({ }^{7} \mathrm{~F}\right) 5 \mathrm{~d}^{2} 6 \mathrm{~s}{ }^{10} \mathrm{I}$ & 1.435 & & \\
\hline & 14346 & & 21.6 & $4 \mathrm{f}^{8}\left({ }^{7} \mathrm{~F}\right) 5 \mathrm{~d}^{2} 6 \mathrm{~s}{ }^{10} \mathrm{P}$ & 12.1 & $4 f^{8}\left({ }^{7} F\right) 5 d^{2} 6 s^{8} S$ & 1.812 & & \\
\hline & 15641 & & 61.8 & $4 \mathrm{f}^{8}\left({ }^{7} \mathrm{~F}\right) 5 \mathrm{~d}^{2} 6 \mathrm{~s}{ }^{10} \mathrm{I}$ & 25.8 & $4 \mathrm{f}^{8}\left({ }^{7} \mathrm{~F}\right) 5 \mathrm{~d}^{2} 6 \mathrm{~s}{ }^{10} \mathrm{H}$ & 1.070 & & \\
\hline & 16070 & & 25.1 & $4 \mathrm{f}^{8}\left({ }^{7} \mathrm{~F}\right) 5 \mathrm{~d}^{2} 6 \mathrm{~s}^{8} \mathrm{~F}$ & 11.4 & $4 \mathrm{f}^{8}\left({ }^{7} \mathrm{~F}\right) 5 \mathrm{~d}^{2} 6 \mathrm{~s}^{8} \mathrm{D}$ & 1.603 & & \\
\hline & 16935 & & 41.9 & $4 f^{8}\left({ }^{7} F\right) 5 d^{2} 6 s{ }^{10} D$ & 23.7 & $4 \mathrm{f}^{8}\left({ }^{7} \mathrm{~F}\right) 5 \mathrm{~d}^{2} 6 \mathrm{~s}{ }^{10} \mathrm{D}$ & 2.072 & & \\
\hline & 17287 & & 52.7 & $4 \mathrm{f}^{8}\left({ }^{7} \mathrm{~F}\right) 5 \mathrm{~d}^{2} 6 \mathrm{~s}{ }^{10} \mathrm{D}$ & 12.5 & $4 \mathrm{f}^{8}\left({ }^{7} \mathrm{~F}\right) 5 \mathrm{~d}^{2} 6 \mathrm{~s}{ }^{10} \mathrm{D}$ & 1.851 & & \\
\hline & 17315 & & 33.3 & $4 f^{8}\left({ }^{7} F\right) 5 d^{2} 6 s^{8} H$ & 13.9 & $4 \mathrm{f}^{8}\left({ }^{7} \mathrm{~F}\right) 5 \mathrm{~d}^{2} 6 \mathrm{~s}^{8} \mathrm{I}$ & 1.262 & & \\
\hline & 18343 & & 11.8 & $4 \mathrm{f}^{8}\left({ }^{5} \mathrm{D}\right) 5 \mathrm{~d} 6 \mathrm{~s}^{2}{ }^{6} \mathrm{D}$ & 8.4 & $4 \mathrm{f}^{8}\left({ }^{5} \mathrm{D}\right) 5 \mathrm{~d} 6 \mathrm{~s}^{2}{ }^{6} \mathrm{D}$ & 1.640 & & \\
\hline & 18432 & & 11.4 & $4 \mathrm{f}^{8}\left({ }^{5} \mathrm{D}\right) 5 \mathrm{~d} 6 \mathrm{~s}^{2}{ }^{6} \mathrm{D}$ & 8.9 & $4 \mathrm{f}^{8}\left({ }^{7} \mathrm{~F}\right) 5 \mathrm{~d}^{2} 6 \mathrm{~s}^{8} \mathrm{P}$ & 1.639 & & \\
\hline & 18719 & & 43.5 & $4 \mathrm{f}^{8}\left({ }^{7} \mathrm{~F}\right) 5 \mathrm{~d}^{2} 6 \mathrm{~s}^{8} \mathrm{I}$ & 16.6 & $4 \mathrm{f}^{8}\left({ }^{7} \mathrm{~F}\right) 5 \mathrm{~d}^{2} 6 \mathrm{~s}^{8} \mathrm{H}$ & 0.877 & & \\
\hline & 18815 & & 22.8 & $4 \mathrm{f}^{8}\left({ }^{7} \mathrm{~F}\right) 5 \mathrm{~d}^{2} 6 \mathrm{~s}{ }^{6} \mathrm{~F}$ & 14.6 & $4 \mathrm{f}^{8}\left({ }^{7} \mathrm{~F}\right) 5 \mathrm{~d}^{2} 6 \mathrm{~s}{ }^{6} \mathrm{D}$ & 1.384 & & \\
\hline & 19132 & & 75.4 & $4 \mathrm{f}^{8}\left({ }^{7} \mathrm{~F}\right) 5 \mathrm{~d}^{2} 6 \mathrm{~s}{ }^{10} \mathrm{G}$ & 9.1 & $4 \mathrm{f}^{8}\left({ }^{7} \mathrm{~F}\right) 5 \mathrm{~d}^{2} 6 \mathrm{~s}{ }^{10} \mathrm{G}$ & 1.638 & & \\
\hline & 19601 & & 41.8 & $4 \mathrm{f}^{8}\left({ }^{7} \mathrm{~F}\right) 5 \mathrm{~d}^{2} 6 \mathrm{~s}^{8} \mathrm{D}$ & 17.0 & $4 \mathrm{f}^{8}\left({ }^{7} \mathrm{~F}\right) 5 \mathrm{~d}^{2} 6 \mathrm{~s}^{8} \mathrm{D}$ & 1.718 & & \\
\hline & 19928 & & 19.9 & $4 f^{8}\left({ }^{7} F\right) 5 d^{2} 6 s{ }^{6} G$ & 12.1 & $4 \mathrm{f}^{8}\left({ }^{7} \mathrm{~F}\right) 5 \mathrm{~d}^{2} 6 \mathrm{~s}^{8} \mathrm{D}$ & 1.398 & & \\
\hline & 20184 & & 14.3 & $4 \mathrm{f}^{8}\left({ }^{7} \mathrm{~F}\right) 5 \mathrm{~d}^{2} 6 \mathrm{~s}{ }^{6} \mathrm{P}$ & 10.6 & $4 \mathrm{f}^{8}\left({ }^{7} \mathrm{~F}\right) 5 \mathrm{~d}^{2} 6 \mathrm{~s}{ }^{6} \mathrm{D}$ & 1.497 & & \\
\hline & 20849 & & 13.4 & $4 f^{8}\left({ }^{7} F\right) 5 d^{2} 6 s^{8} G$ & 9.0 & $4 \mathrm{f}^{8}\left({ }^{7} \mathrm{~F}\right) 5 \mathrm{~d}^{2} 6 \mathrm{~s}^{8} \mathrm{~F}$ & 1.567 & & \\
\hline \multicolumn{10}{|l|}{$J=9 / 2$} \\
\hline 1371.045 & 1332 & 39 & 31.5 & $4 f^{8}\left({ }^{7} F\right) 5 d 6 s^{2}{ }^{8} G$ & 29.5 & $4 \mathrm{f}^{8}\left({ }^{7} \mathrm{~F}\right) 5 \mathrm{~d} 6 \mathrm{~s}^{2}{ }^{8} \mathrm{~F}$ & 1.545 & 1.541 & -0.004 \\
\hline 2840.170 & 2864 & -23 & 43.9 & $4 \mathrm{f}^{8}\left({ }^{7} \mathrm{~F}\right) 5 \mathrm{~d} 6 \mathrm{~s}^{2}{ }^{8} \mathrm{G}$ & 36.1 & $4 \mathrm{f}^{8}\left({ }^{7} \mathrm{~F}\right) 5 \mathrm{~d} 6 \mathrm{~s}^{2}{ }^{8} \mathrm{D}$ & 1.541 & 1.544 & 0.002 \\
\hline
\end{tabular}


Table 1. Continued.

\begin{tabular}{|c|c|c|c|c|c|c|c|c|c|}
\hline$E_{\exp }$ & $E_{\text {calc }}$ & $\Delta E$ & $\%$ & Main comp. & $\%$ & Sec. comp. & $g_{J \text { calc }}$ & $g_{J \exp }$ & $\Delta g_{J}$ \\
\hline 5829.860 & 5780 & 50 & 50.7 & $4 \mathrm{f}^{8}\left({ }^{7} \mathrm{~F}\right) 5 \mathrm{~d} 6 \mathrm{~s}^{2}{ }^{8} \mathrm{~F}$ & 14.5 & $4 \mathrm{f}^{8}\left({ }^{7} \mathrm{~F}\right) 5 \mathrm{~d} 6 \mathrm{~s}^{2}{ }^{8} \mathrm{P}$ & 1.578 & 1.580 & 0.002 \\
\hline 7441.030 & 7398 & 43 & 66.4 & $4 \mathrm{f}^{8}\left({ }^{7} \mathrm{~F}\right) 5 \mathrm{~d} 6 \mathrm{~s}^{2}{ }^{8} \mathrm{H}$ & 9.5 & $4 \mathrm{f}^{8}\left({ }^{7} \mathrm{~F}\right) 5 \mathrm{~d} 6 \mathrm{~s}^{2}{ }^{6} \mathrm{G}$ & 1.247 & 1.240 & -0.007 \\
\hline 7824.190 & 7874 & -49 & 60.0 & $4 \mathrm{f}^{8}\left({ }^{7} \mathrm{~F}\right) 5 \mathrm{~d} 6 \mathrm{~s}^{2}{ }^{6} \mathrm{~F}$ & 11.1 & $4 \mathrm{f}^{8}\left({ }^{7} \mathrm{~F}\right) 5 \mathrm{~d} 6 \mathrm{~s}^{2}{ }^{6} \mathrm{D}$ & 1.413 & 1.433 & 0.020 \\
\hline 8097.875 & 8103 & -5 & 61.1 & $4 \mathrm{f}^{8}\left({ }^{7} \mathrm{~F}\right) 5 \mathrm{~d} 6 \mathrm{~s}^{2}{ }^{8} \mathrm{P}$ & 19.5 & $4 \mathrm{f}^{8}\left({ }^{7} \mathrm{~F}\right) 5 \mathrm{~d} 6 \mathrm{~s}^{2}{ }^{8} \mathrm{D}$ & 1.727 & 1.750 & 0.023 \\
\hline 9145.230 & 9118 & 27 & 46.3 & $4 \mathrm{f}^{8}\left({ }^{7} \mathrm{~F}\right) 5 \mathrm{~d}^{2} 6 \mathrm{~s}{ }^{10} \mathrm{G}$ & 19.6 & $4 \mathrm{f}^{8}\left({ }^{7} \mathrm{~F}\right) 5 \mathrm{~d}^{2} 6 \mathrm{~s}{ }^{10} \mathrm{~F}$ & 1.673 & 1.670 & -0.003 \\
\hline 9897.730 & 9886 & 12 & 26.5 & $4 \mathrm{f}^{8}\left({ }^{7} \mathrm{~F}\right) 5 \mathrm{~d}^{2} 6 \mathrm{~s}{ }^{10} \mathrm{~S}$ & 19.8 & $4 \mathrm{f}^{8}\left({ }^{7} \mathrm{~F}\right) 5 \mathrm{~d} 6 \mathrm{~s}^{2}{ }^{6} \mathrm{G}$ & 1.668 & 1.810 & 0.142 \\
\hline 9986.73 & 9934 & 53 & 29.7 & $4 f^{8}\left({ }^{7} F\right) 5 d 6 s^{2}{ }^{6} G$ & 18.8 & $4 \mathrm{f}^{8}\left({ }^{7} \mathrm{~F}\right) 5 \mathrm{~d}^{2} 6 \mathrm{~s}{ }^{10} \mathrm{~S}$ & 1.567 & & \\
\hline 10680.17 & 10662 & 19 & 49.1 & $4 \mathrm{f}^{8}\left({ }^{7} \mathrm{~F}\right) 5 \mathrm{~d} 6 \mathrm{~s}^{2}{ }^{6} \mathrm{D}$ & 19.6 & $4 \mathrm{f}^{8}\left({ }^{7} \mathrm{~F}\right) 5 \mathrm{~d} 6 \mathrm{~s}^{2}{ }^{6} \mathrm{~F}$ & 1.474 & & \\
\hline 11956.255 & 11943 & 14 & 72.6 & $4 \mathrm{f}^{8}\left({ }^{7} \mathrm{~F}\right) 5 \mathrm{~d} 6 \mathrm{~s}^{2}{ }^{6} \mathrm{H}$ & 6.8 & $4 \mathrm{f}^{8}\left({ }^{7} \mathrm{~F}\right) 5 \mathrm{~d} 6 \mathrm{~s}^{2}{ }^{8} \mathrm{H}$ & 1.104 & & \\
\hline 12228.28 & 12270 & -42 & 36.8 & $4 \mathrm{f}^{8}\left({ }^{7} \mathrm{~F}\right) 5 \mathrm{~d}^{2} 6 \mathrm{~s}{ }^{10} \mathrm{~F}$ & 15.5 & $4 \mathrm{f}^{8}\left({ }^{7} \mathrm{~F}\right) 5 \mathrm{~d}^{2} 6 \mathrm{~s}{ }^{10} \mathrm{D}$ & 1.727 & & \\
\hline 12776.31 & 12790 & -13 & 29.0 & $4 \mathrm{f}^{8}\left({ }^{7} \mathrm{~F}\right) 5 \mathrm{~d}^{2} 6 \mathrm{~s}^{8} \mathrm{G}$ & 15.8 & $4 \mathrm{f}^{8}\left({ }^{7} \mathrm{~F}\right) 5 \mathrm{~d}^{2} 6 \mathrm{~s}^{8} \mathrm{~F}$ & 1.468 & & \\
\hline \multirow[t]{19}{*}{13751.41} & 13785 & -33 & 46.1 & $4 \mathrm{f}^{8}\left({ }^{7} \mathrm{~F}\right) 5 \mathrm{~d}^{2} 6 \mathrm{~s}{ }^{10} \mathrm{H}$ & 28.3 & $4 \mathrm{f}^{8}\left({ }^{7} \mathrm{~F}\right) 5 \mathrm{~d}^{2} 6 \mathrm{~s}{ }^{10} \mathrm{I}$ & 1.364 & & \\
\hline & 14709 & & 25.7 & $4 \mathrm{f}^{8}\left({ }^{7} \mathrm{~F}\right) 5 \mathrm{~d}^{2} 6 \mathrm{~s}{ }^{10} \mathrm{P}$ & 22.5 & $4 \mathrm{f}^{8}\left({ }^{7} \mathrm{~F}\right) 5 \mathrm{~d}^{2} 6 \mathrm{~s}{ }^{10} \mathrm{~S}$ & 1.850 & & \\
\hline & 15141 & & 20.5 & $4 \mathrm{f}^{8}\left({ }^{7} \mathrm{~F}\right) 5 \mathrm{~d}^{2} 6 \mathrm{~s}^{8} \mathrm{D}$ & 14.1 & $4 \mathrm{f}^{8}\left({ }^{7} \mathrm{~F}\right) 5 \mathrm{~d}^{2} 6 \mathrm{~s}^{8} \mathrm{P}$ & 1.582 & & \\
\hline & 15537 & & 54.0 & $4 \mathrm{f}^{8}\left({ }^{7} \mathrm{~F}\right) 5 \mathrm{~d}^{2} 6 \mathrm{~s}{ }^{10} \mathrm{I}$ & 29.9 & $4 \mathrm{f}^{8}\left({ }^{7} \mathrm{~F}\right) 5 \mathrm{~d}^{2} 6 \mathrm{~s}{ }^{10} \mathrm{H}$ & 1.244 & & \\
\hline & 16025 & & 85.4 & $4 f^{8}\left({ }^{7} \mathrm{~F}\right) 5 \mathrm{~d}^{2} 6 \mathrm{~s}{ }^{10} \mathrm{D}$ & 3.7 & $4 \mathrm{f}^{8}\left({ }^{7} \mathrm{~F}\right) 5 \mathrm{~d}^{2} 6 \mathrm{~s}^{8} \mathrm{D}$ & 1.854 & & \\
\hline & 16715 & & 30.6 & $4 f^{8}\left({ }^{7} \mathrm{~F}\right) 5 \mathrm{~d}^{2} 6 \mathrm{~s}^{8} \mathrm{H}$ & 13.5 & $4 \mathrm{f}^{8}\left({ }^{7} \mathrm{~F}\right) 5 \mathrm{~d}^{2} 6 \mathrm{~s}^{8} \mathrm{P}$ & 1.333 & & \\
\hline & 17314 & & 18.5 & $4 \mathrm{f}^{8}\left({ }^{7} \mathrm{~F}\right) 5 \mathrm{~d}^{2} 6 \mathrm{~s}^{8} \mathrm{P}$ & 16.2 & $4 \mathrm{f}^{8}\left({ }^{7} \mathrm{~F}\right) 5 \mathrm{~d}^{2} 6 \mathrm{~s}^{8} \mathrm{~F}$ & 1.509 & & \\
\hline & 17631 & & 47.0 & $4 \mathrm{f}^{8}\left({ }^{7} \mathrm{~F}\right) 5 \mathrm{~d}^{2} 6 \mathrm{~s}{ }^{10} \mathrm{D}$ & 28.4 & $4 \mathrm{f}^{8}\left({ }^{7} \mathrm{~F}\right) 5 \mathrm{~d}^{2} 6 \mathrm{~s}{ }^{10} \mathrm{P}$ & 1.876 & & \\
\hline & 18251 & & 21.9 & $4 \mathrm{f}^{8}\left({ }^{5} \mathrm{D}\right) 5 \mathrm{~d} 6 \mathrm{~s}^{2}{ }^{6} \mathrm{~F}$ & 18.1 & $4 \mathrm{f}^{8}\left({ }^{5} \mathrm{D}\right) 5 \mathrm{~d} 6 \mathrm{~s}^{2}{ }^{6} \mathrm{D}$ & 1.459 & & \\
\hline & 18307 & & 47.3 & $4 \mathrm{f}^{8}\left({ }^{7} \mathrm{~F}\right) 5 \mathrm{~d}^{2} 6 \mathrm{~s}^{8} \mathrm{D}$ & 20.0 & $4 f^{8}\left({ }^{7} F\right) 5 d^{2} 6 s^{8} D$ & 1.661 & & \\
\hline & 18470 & & 20.8 & $4 \mathrm{f}^{8}\left({ }^{7} \mathrm{~F}\right) 5 \mathrm{~d}^{2} 6 \mathrm{~s}^{8} \mathrm{I}$ & 13.1 & $4 f^{8}\left({ }^{7} F\right) 5 d^{2} 6 s{ }^{6} G$ & 1.212 & & \\
\hline & 18611 & & 46.2 & $4 f^{8}\left({ }^{7} F\right) 5 d^{2} 6 s{ }^{10} G$ & 6.6 & $4 \mathrm{f}^{8}\left({ }^{7} \mathrm{~F}\right) 5 \mathrm{~d}^{2} 6 \mathrm{~s}{ }^{10} \mathrm{G}$ & 1.474 & & \\
\hline & 18643 & & 25.3 & $4 f^{8}\left({ }^{7} F\right) 5 d^{2} 6 s{ }^{10} G$ & 17.5 & $4 \mathrm{f}^{8}\left({ }^{7} \mathrm{~F}\right) 5 \mathrm{~d}^{2} 6 \mathrm{~s}^{8} \mathrm{I}$ & 1.315 & & \\
\hline & 19274 & & 12.0 & $4 \mathrm{f}^{8}\left({ }^{7} \mathrm{~F}\right) 5 \mathrm{~d}^{2} 6 \mathrm{~s}^{8} \mathrm{P}$ & 8.1 & $4 \mathrm{f}^{8}\left({ }^{7} \mathrm{~F}\right) 5 \mathrm{~d}^{2} 6 \mathrm{~s}{ }^{6} \mathrm{H}$ & 1.468 & & \\
\hline & 19632 & & 26.8 & $4 \mathrm{f}^{8}\left({ }^{7} \mathrm{~F}\right) 5 \mathrm{~d}^{3}{ }^{10} \mathrm{D}$ & 16.6 & $4 \mathrm{f}^{8}\left({ }^{7} \mathrm{~F}\right) 5 \mathrm{~d}^{3}{ }^{10} \mathrm{P}$ & 1.740 & & \\
\hline & 19858 & & 13.0 & $4 \mathrm{f}^{8}\left({ }^{7} \mathrm{~F}\right) 5 \mathrm{~d}^{2} 6 \mathrm{~s}^{8} \mathrm{P}$ & 11.5 & $4 \mathrm{f}^{8}\left({ }^{7} \mathrm{~F}\right) 5 \mathrm{~d}^{3}{ }^{10} \mathrm{D}$ & 1.616 & & \\
\hline & 20199 & & 17.8 & $4 f^{8}\left({ }^{7} F\right) 5 d^{2} 6 s^{8} D$ & 14.6 & $4 \mathrm{f}^{8}\left({ }^{7} \mathrm{~F}\right) 5 \mathrm{~d}^{2} 6 \mathrm{~s}^{8} \mathrm{P}$ & 1.605 & & \\
\hline & 20563 & & 12.5 & $4 \mathrm{f}^{8}\left({ }^{7} \mathrm{~F}\right) 5 \mathrm{~d}^{2} 6 \mathrm{~s}{ }^{6} \mathrm{H}$ & 11.6 & $4 \mathrm{f}^{8}\left({ }^{7} \mathrm{~F}\right) 5 \mathrm{~d}^{2} 6 \mathrm{~s}^{8} \mathrm{G}$ & 1.370 & & \\
\hline & 20583 & & 24.6 & $4 f^{8}\left({ }^{7} F\right) 5 d^{2} 6 s{ }^{8} D$ & 15.3 & $4 \mathrm{f}^{8}\left({ }^{7} \mathrm{~F}\right) 5 \mathrm{~d}^{2} 6 \mathrm{~s}^{8} \mathrm{P}$ & 1.636 & & \\
\hline \multicolumn{10}{|l|}{$J=11 / 2$} \\
\hline 509.845 & 476 & 34 & 32.6 & $4 \mathrm{f}^{8}\left({ }^{7} \mathrm{~F}\right) 5 \mathrm{~d} 6 \mathrm{~s}^{2}{ }^{8} \mathrm{G}$ & 31.1 & $4 \mathrm{f}^{8}\left({ }^{7} \mathrm{~F}\right) 5 \mathrm{~d} 6 \mathrm{~s}^{2}{ }^{8} \mathrm{~F}$ & 1.519 & 1.517 & -0.002 \\
\hline 2310.090 & 2336 & -26 & 45.7 & $4 f^{8}\left({ }^{7} \mathrm{~F}\right) 5 \mathrm{~d} 6 \mathrm{~s}^{2}{ }^{8} \mathrm{D}$ & 40.5 & $4 \mathrm{f}^{8}\left({ }^{7} \mathrm{~F}\right) 5 \mathrm{~d} 6 \mathrm{~s}^{2}{ }^{8} \mathrm{G}$ & 1.529 & 1.530 & 0.002 \\
\hline 5353.370 & 5334 & 19 & 50.4 & $4 \mathrm{f}^{8}\left({ }^{7} \mathrm{~F}\right) 5 \mathrm{~d} 6 \mathrm{~s}^{2}{ }^{8} \mathrm{~F}$ & 21.7 & $4 \mathrm{f}^{8}\left({ }^{7} \mathrm{~F}\right) 5 \mathrm{~d} 6 \mathrm{~s}^{2}{ }^{8} \mathrm{D}$ & 1.534 & 1.545 & 0.011 \\
\hline 6674.155 & 6700 & -26 & 51.2 & $4 \mathrm{f}^{8}\left({ }^{7} \mathrm{~F}\right) 5 \mathrm{~d} 6 \mathrm{~s}^{2}{ }^{6} \mathrm{~F}$ & 18.3 & $4 \mathrm{f}^{8}\left({ }^{7} \mathrm{~F}\right) 5 \mathrm{~d} 6 \mathrm{~s}^{2}{ }^{6} \mathrm{G}$ & 1.415 & 1.320 & -0.095 \\
\hline 6988.820 & 6996 & -7 & 60.8 & $4 \mathrm{f}^{8}\left({ }^{7} \mathrm{~F}\right) 5 \mathrm{~d} 6 \mathrm{~s}^{2}{ }^{8} \mathrm{H}$ & 18.0 & $4 \mathrm{f}^{8}\left({ }^{7} \mathrm{~F}\right) 5 \mathrm{~d} 6 \mathrm{~s}^{2}{ }^{6} \mathrm{~F}$ & 1.330 & 1.315 & -0.015 \\
\hline 8646.210 & 8623 & 24 & 53.2 & $4 f^{8}\left({ }^{7} F\right) 5 d^{2} 6 s{ }^{10} G$ & 21.3 & $4 \mathrm{f}^{8}\left({ }^{7} \mathrm{~F}\right) 5 \mathrm{~d}^{2} 6 \mathrm{~s}{ }^{10} \mathrm{~F}$ & 1.586 & 1.600 & 0.014 \\
\hline 8932.120 & 8905 & 27 & 54.0 & $4 \mathrm{f}^{8}\left({ }^{7} \mathrm{~F}\right) 5 \mathrm{~d} 6 \mathrm{~s}^{2}{ }^{6} \mathrm{G}$ & 14.8 & $4 \mathrm{f}^{8}\left({ }^{7} \mathrm{~F}\right) 5 \mathrm{~d} 6 \mathrm{~s}^{2}{ }^{6} \mathrm{~F}$ & 1.347 & 1.470 & 0.123 \\
\hline 10997.850 & 10967 & 31 & 70.9 & $4 \mathrm{f}^{8}\left({ }^{7} \mathrm{~F}\right) 5 \mathrm{~d} 6 \mathrm{~s}^{2}{ }^{6} \mathrm{H}$ & 9.9 & $4 \mathrm{f}^{8}\left({ }^{7} \mathrm{~F}\right) 5 \mathrm{~d} 6 \mathrm{~s}^{2}{ }^{8} \mathrm{H}$ & 1.224 & 1.210 & -0.014 \\
\hline 11260.41 & 11303 & -43 & 25.3 & $4 f^{8}\left({ }^{7} \mathrm{~F}\right) 5 \mathrm{~d}^{2} 6 \mathrm{~s}{ }^{10} \mathrm{D}$ & 20.0 & $4 \mathrm{f}^{8}\left({ }^{7} \mathrm{~F}\right) 5 \mathrm{~d}^{2} 6 \mathrm{~s}{ }^{10} \mathrm{P}$ & 1.669 & 1.680 & 0.011 \\
\hline 12453.14 & 12471 & -18 & 28.3 & $4 f^{8}\left({ }^{7} F\right) 5 d^{2} 6 s{ }^{8} G$ & 15.7 & $4 \mathrm{f}^{8}\left({ }^{7} \mathrm{~F}\right) 5 \mathrm{~d}^{2} 6 \mathrm{~s}^{8} \mathrm{G}$ & 1.474 & & \\
\hline 13071.30 & 13082 & -11 & 28.4 & $4 \mathrm{f}^{8}\left({ }^{7} \mathrm{~F}\right) 5 \mathrm{~d}^{2} 6 \mathrm{~s}{ }^{10} \mathrm{H}$ & 27.6 & $4 \mathrm{f}^{8}\left({ }^{7} \mathrm{~F}\right) 5 \mathrm{~d}^{2} 6 \mathrm{~s}{ }^{10} \mathrm{P}$ & 1.564 & & \\
\hline \multirow[t]{3}{*}{13666.46} & 13678 & -11 & 19.9 & $4 \mathrm{f}^{8}\left({ }^{7} \mathrm{~F}\right) 5 \mathrm{~d}^{2} 6 \mathrm{~s}{ }^{10} \mathrm{I}$ & 18.7 & $4 \mathrm{f}^{8}\left({ }^{7} \mathrm{~F}\right) 5 \mathrm{~d}^{2} 6 \mathrm{~s}{ }^{10} \mathrm{P}$ & 1.538 & & \\
\hline & 14597 & & 78.4 & $4 \mathrm{f}^{8}\left({ }^{7} \mathrm{~F}\right) 5 \mathrm{~d}^{2} 6 \mathrm{~s}{ }^{10} \mathrm{D}$ & 5.7 & $4 \mathrm{f}^{8}\left({ }^{7} \mathrm{~F}\right) 5 \mathrm{~d}^{2} 6 \mathrm{~s}{ }^{10} \mathrm{~F}$ & 1.734 & & \\
\hline & 15249 & & 21.9 & $4 \mathrm{f}^{8}\left({ }^{7} \mathrm{~F}\right) 5 \mathrm{~d}^{2} 6 \mathrm{~s}^{8} \mathrm{~F}$ & 12.2 & $4 \mathrm{f}^{8}\left({ }^{7} \mathrm{~F}\right) 5 \mathrm{~d}^{2} 6 \mathrm{~s}^{8} \mathrm{H}$ & 1.498 & & \\
\hline
\end{tabular}


Table 1. Continued.

\begin{tabular}{|c|c|c|c|c|c|c|c|c|c|}
\hline$E_{\text {exp }}$ & $E_{\text {calc }}$ & $\Delta E$ & $\%$ & Main comp. & $\%$ & Sec. comp. & $g_{J \text { calc }}$ & $g_{J \exp }$ & $\Delta g_{J}$ \\
\hline & 15335 & & 49.4 & $4 f^{8}\left({ }^{7} \mathrm{~F}\right) 5 \mathrm{~d}^{2} 6 \mathrm{~s}{ }^{10} \mathrm{I}$ & 31.8 & $4 \mathrm{f}^{8}\left({ }^{7} \mathrm{~F}\right) 5 \mathrm{~d}^{2} 6 \mathrm{~s}{ }^{10} \mathrm{H}$ & 1.323 & & \\
\hline & 16439 & & 22.0 & $4 \mathrm{f}^{8}\left({ }^{7} \mathrm{~F}\right) 5 \mathrm{~d}^{2} 6 \mathrm{~s}^{8} \mathrm{H}$ & 14.5 & $4 \mathrm{f}^{8}\left({ }^{7} \mathrm{~F}\right) 5 \mathrm{~d}^{2} 6 \mathrm{~s}^{8} \mathrm{I}$ & 1.367 & & \\
\hline & 16920 & & 27.9 & $4 f^{8}\left({ }^{7} \mathrm{~F}\right) 5 \mathrm{~d}^{2} 6 \mathrm{~s}^{8} \mathrm{D}$ & 18.5 & $4 \mathrm{f}^{8}\left({ }^{7} \mathrm{~F}\right) 5 \mathrm{~d}^{2} 6 \mathrm{~s}^{8} \mathrm{D}$ & 1.553 & & \\
\hline & 17031 & & 54.4 & $4 \mathrm{f}^{8}\left({ }^{7} \mathrm{~F}\right) 5 \mathrm{~d}^{2} 6 \mathrm{~s}{ }^{10} \mathrm{D}$ & 21.1 & $4 \mathrm{f}^{8}\left({ }^{7} \mathrm{~F}\right) 5 \mathrm{~d}^{2} 6 \mathrm{~s}{ }^{10} \mathrm{P}$ & 1.744 & & \\
\hline & 17988 & & 65.4 & $4 \mathrm{f}^{8}\left({ }^{7} \mathrm{~F}\right) 5 \mathrm{~d}^{2} 6 \mathrm{~s}{ }^{10} \mathrm{G}$ & 9.1 & $4 \mathrm{f}^{8}\left({ }^{7} \mathrm{~F}\right) 5 \mathrm{~d}^{2} 6 \mathrm{~s}{ }^{10} \mathrm{G}$ & 1.541 & & \\
\hline & 18145 & & 18.5 & $4 \mathrm{f}^{8}\left({ }^{7} \mathrm{~F}\right) 5 \mathrm{~d}^{2} 6 \mathrm{~s}^{8} \mathrm{I}$ & 18.3 & $4 f^{8}\left({ }^{7} \mathrm{~F}\right) 5 \mathrm{~d}^{2} 6 \mathrm{~s}{ }^{6} \mathrm{H}$ & 1.260 & & \\
\hline & 18337 & & 21.8 & $4 \mathrm{f}^{8}\left({ }^{7} \mathrm{~F}\right) 5 \mathrm{~d}^{2} 6 \mathrm{~s}^{8} \mathrm{H}$ & 17.9 & $4 \mathrm{f}^{8}\left({ }^{7} \mathrm{~F}\right) 5 \mathrm{~d}^{2} 6 \mathrm{~s}^{8} \mathrm{I}$ & 1.256 & & \\
\hline & 18793 & & 36.3 & $4 \mathrm{f}^{9}\left({ }^{6} \mathrm{H}\right) 6 \mathrm{~s} 6 \mathrm{p}{ }^{4} \mathrm{G}$ & 9.0 & $4 \mathrm{f}^{9}\left({ }^{6} \mathrm{H}\right) 6 \mathrm{~s} 6 \mathrm{p}{ }^{6} \mathrm{G}$ & 1.325 & & \\
\hline & 18922 & & 24.9 & $4 f^{8}\left({ }^{7} F\right) 5 d^{2} 6 s^{8} D$ & 8.9 & $4 \mathrm{f}^{8}\left({ }^{7} \mathrm{~F}\right) 5 \mathrm{~d}^{2} 6 \mathrm{~s}{ }^{6} \mathrm{~F}$ & 1.474 & & \\
\hline & 19235 & & 14.0 & $4 \mathrm{f}^{8}\left({ }^{7} \mathrm{~F}\right) 5 \mathrm{~d}^{2} 6 \mathrm{~s}^{8} \mathrm{D}$ & 10.9 & $4 \mathrm{f}^{8}\left({ }^{7} \mathrm{~F}\right) 5 \mathrm{~d}^{2} 6 \mathrm{~s}^{8} \mathrm{~F}$ & 1.455 & & \\
\hline & 19361 & & 22.5 & $4 \mathrm{f}^{8}\left({ }^{5} \mathrm{D}\right) 5 \mathrm{~d} 6 \mathrm{~s}^{2}{ }^{6} \mathrm{~F}$ & 18.3 & $4 \mathrm{f}^{8}\left({ }^{5} \mathrm{D}\right) 5 \mathrm{~d} 6 \mathrm{~s}^{2}{ }^{6} \mathrm{~F}$ & 1.413 & & \\
\hline & 19463 & & 42.5 & $4 \mathrm{f}^{8}\left({ }^{7} \mathrm{~F}\right) 5 \mathrm{~d}^{3}{ }^{10} \mathrm{D}$ & 25.3 & $4 \mathrm{f}^{8}\left({ }^{7} \mathrm{~F}\right) 5 \mathrm{~d}^{3}{ }^{10} \mathrm{~F}$ & 1.714 & & \\
\hline & 19736 & & 9.1 & $4 \mathrm{f}^{8}\left({ }^{5} \mathrm{D}\right) 5 \mathrm{~d} 6 \mathrm{~s}^{2}{ }^{6} \mathrm{~F}$ & 7.5 & $4 \mathrm{f}^{8}\left({ }^{5} \mathrm{D}\right) 5 \mathrm{~d} 6 \mathrm{~s}^{2}{ }^{6} \mathrm{~F}$ & 1.364 & & \\
\hline & 19780 & & 8.5 & $4 \mathrm{f}^{9}\left({ }^{6} \mathrm{H}\right) 6 \mathrm{~s} 6 \mathrm{p}{ }^{8} \mathrm{H}$ & 7.9 & $4 f^{8}\left({ }^{7} F\right) 5 d^{2} 6 s{ }^{8} G$ & 1.377 & & \\
\hline & 20043 & & 15.4 & $4 \mathrm{f}^{8}\left({ }^{7} \mathrm{~F}\right) 5 \mathrm{~d}^{2} 6 \mathrm{~s}^{8} \mathrm{~F}$ & 8.4 & $4 f^{8}\left({ }^{7} \mathrm{~F}\right) 5 \mathrm{~d}^{2} 6 \mathrm{~s}^{8} \mathrm{D}$ & 1.465 & & \\
\hline & 20401 & & 18.5 & $4 f^{8}\left({ }^{7} F\right) 5 d^{2} 6 s^{8} D$ & 10.9 & $4 f^{8}\left({ }^{7} F\right) 5 d^{2} 6 s{ }^{6} G$ & 1.384 & & \\
\hline & 20558 & & 35.8 & $4 f^{8}\left({ }^{7} F\right) 5 d^{2} 6 s^{8} G$ & 8.2 & $4 f^{8}\left({ }^{7} F\right) 5 d^{2} 6 s{ }^{6} G$ & 1.416 & & \\
\hline & 20924 & & 19.5 & $4 \mathrm{f}^{9}\left({ }^{6} \mathrm{H}\right) 6 \mathrm{~s} 6 \mathrm{p}{ }^{6} \mathrm{G}$ & 11.8 & $4 \mathrm{f}^{9}\left({ }^{6} \mathrm{H}\right) 6 \mathrm{~s} 6 \mathrm{p}{ }^{8} \mathrm{H}$ & 1.310 & & \\
\hline & 21069 & & 17.6 & $4 \mathrm{f}^{8}\left({ }^{7} \mathrm{~F}\right) 5 \mathrm{~d}^{2} 6 \mathrm{~s}{ }^{6} \mathrm{I}$ & 11.8 & $4 f^{8}\left({ }^{7} \mathrm{~F}\right) 5 \mathrm{~d}^{2} 6 \mathrm{~s}^{8} \mathrm{D}$ & 1.268 & & \\
\hline & 21159 & & 9.0 & $4 f^{8}\left({ }^{7} \mathrm{~F}\right) 5 \mathrm{~d}^{2} 6 \mathrm{~s}^{8} \mathrm{H}$ & 8.9 & $4 \mathrm{f}^{8}\left({ }^{7} \mathrm{~F}\right) 5 \mathrm{~d}^{2} 6 \mathrm{~s}{ }^{6} \mathrm{H}$ & 1.354 & & \\
\hline & 21523 & & 7.6 & $4 f^{8}\left({ }^{7} \mathrm{~F}\right) 5 \mathrm{~d}^{2} 6 \mathrm{~s}^{8} \mathrm{D}$ & 7.1 & $4 f^{8}\left({ }^{7} \mathrm{~F}\right) 5 \mathrm{~d}^{2} 6 \mathrm{~s}^{8} \mathrm{H}$ & 1.404 & & \\
\hline & 21717 & & 17.8 & $4 \mathrm{f}^{9}\left({ }^{6} \mathrm{H}\right) 6 \mathrm{~s} 6 \mathrm{p}{ }^{8} \mathrm{G}$ & 8.4 & $4 \mathrm{f}^{9}\left({ }^{6} \mathrm{H}\right) 6 \mathrm{~s} 6 \mathrm{p}{ }^{4} \mathrm{H}$ & 1.240 & & \\
\hline & 21854 & & 17.2 & $4 \mathrm{f}^{9}\left({ }^{6} \mathrm{H}\right) 6 \mathrm{~s} 6 \mathrm{p}^{8} \mathrm{I}$ & 14.0 & $4 \mathrm{f}^{9}\left({ }^{6} \mathrm{H}\right) 6 \mathrm{~s} 6 \mathrm{p}{ }^{6} \mathrm{I}$ & 1.182 & & \\
\hline & 21984 & & 14.5 & $4 \mathrm{f}^{8}\left({ }^{7} \mathrm{~F}\right) 5 \mathrm{~d}^{2} 6 \mathrm{~s}{ }^{10} \mathrm{~F}$ & 6.3 & $4 \mathrm{f}^{8}\left({ }^{7} \mathrm{~F}\right) 5 \mathrm{~d}^{2} 6 \mathrm{~s}{ }^{6} \mathrm{~F}$ & 1.357 & & \\
\hline & 22176 & & 18.0 & $4 \mathrm{f}^{8}\left({ }^{7} \mathrm{~F}\right) 5 \mathrm{~d}^{2} 6 \mathrm{~s}{ }^{10} \mathrm{~F}$ & 4.8 & $4 f^{8}\left({ }^{7} F\right) 5 d^{2} 6 s^{6} I$ & 1.434 & & \\
\hline & 22217 & & 23.0 & $4 \mathrm{f}^{8}\left({ }^{7} \mathrm{~F}\right) 5 \mathrm{~d}^{3}{ }^{10} \mathrm{P}$ & 21.2 & $4 \mathrm{f}^{8}\left({ }^{7} \mathrm{~F}\right) 5 \mathrm{~d}^{3}{ }^{10} \mathrm{~F}$ & 1.649 & & \\
\hline & 22231 & & 11.5 & $4 f^{9}\left({ }^{6} H\right) 6 s 6 p{ }^{4} G$ & 10.1 & $4 \mathrm{f}^{8}\left({ }^{7} \mathrm{~F}\right) 5 \mathrm{~d}^{2} 6 \mathrm{~s}{ }^{10} \mathrm{~F}$ & 1.355 & & \\
\hline & 22442 & & 15.4 & $4 \mathrm{f}^{8}\left({ }^{7} \mathrm{~F}\right) 5 \mathrm{~d}^{2} 6 \mathrm{~s}{ }^{10} \mathrm{~F}$ & 9.3 & $4 f^{8}\left({ }^{7} F\right) 5 d^{2} 6 s^{8} \mathrm{I}$ & 1.404 & & \\
\hline & 22561 & & 13.3 & $4 f^{8}\left({ }^{7} F\right) 5 d^{2} 6 s{ }^{6} F$ & 10.7 & $4 f^{8}\left({ }^{7} F\right) 5 d^{2} 6 s{ }^{6} G$ & 1.348 & & \\
\hline & 22742 & & 31.2 & $4 f^{8}\left({ }^{7} F\right) 5 d^{2} 6 s{ }^{4} H$ & 10.2 & $4 \mathrm{f}^{8}\left({ }^{7} \mathrm{~F}\right) 5 \mathrm{~d}^{2} 6 \mathrm{~s}{ }^{4} \mathrm{G}$ & 1.243 & & \\
\hline & 22797 & & 28.6 & $4 \mathrm{f}^{8}\left({ }^{7} \mathrm{~F}\right) 5 \mathrm{~d}^{2} 6 \mathrm{~s}^{8} \mathrm{D}$ & 17.9 & $4 \mathrm{f}^{8}\left({ }^{7} \mathrm{~F}\right) 5 \mathrm{~d}^{2} 6 \mathrm{~s}^{8} \mathrm{D}$ & 1.526 & & \\
\hline & 23192 & & 10.0 & $4 \mathrm{f}^{8}\left({ }^{7} \mathrm{~F}\right) 5 \mathrm{~d}^{2} 6 \mathrm{~s}{ }^{6} \mathrm{~F}$ & 6.7 & $4 f^{8}\left({ }^{7} F\right) 5 d^{2} 6 s{ }^{6} F$ & 1.354 & & \\
\hline & 23242 & & 7.4 & $4 f^{8}\left({ }^{7} F\right) 5 d^{2} 6 s^{8} G$ & 6.4 & $4 f^{8}\left({ }^{7} F\right) 5 d^{2} 6 s{ }^{6} G$ & 1.315 & & \\
\hline & 23291 & & 8.7 & $4 f^{9}\left({ }^{6} F\right) 6 s 6 p{ }^{8} D$ & 5.3 & $4 \mathrm{f}^{9}\left({ }^{6} \mathrm{H}\right) 6 \mathrm{~s} 6 \mathrm{p}{ }^{8} \mathrm{H}$ & 1.311 & & \\
\hline & 23357 & & 19.7 & $4 f^{9}\left({ }^{6} F\right) 6 s 6 p{ }^{8} D$ & 7.7 & $4 \mathrm{f}^{9}\left({ }^{6} \mathrm{~F}\right) 6 \mathrm{~s} 6 \mathrm{p}{ }^{8} \mathrm{~F}$ & 1.417 & & \\
\hline & 23530 & & 11.2 & $4 f^{8}\left({ }^{7} F\right) 5 d^{2} 6 s{ }^{4} G$ & 9.1 & $4 f^{8}\left({ }^{7} F\right) 5 d^{2} 6 s^{8} G$ & 1.344 & & \\
\hline & 23625 & & 13.4 & $4 \mathrm{f}^{9}\left({ }^{6} \mathrm{H}\right) 6 \mathrm{~s} 6 \mathrm{p}{ }^{8} \mathrm{G}$ & 10.6 & $4 f^{9}\left({ }^{6} \mathrm{H}\right) 6 \mathrm{~s} 6 \mathrm{p}{ }^{8} \mathrm{H}$ & 1.300 & & \\
\hline & 23722 & & 13.9 & $4 \mathrm{f}^{9}\left({ }^{6} \mathrm{~F}\right) 6 \mathrm{~s} 6 \mathrm{p}{ }^{8} \mathrm{D}$ & 13.7 & $4 \mathrm{f}^{9}\left({ }^{6} \mathrm{H}\right) 6 \mathrm{~s} 6 \mathrm{p}{ }^{4} \mathrm{H}$ & 1.321 & & \\
\hline & 23806 & & 40.5 & $4 \mathrm{f}^{8}\left({ }^{7} \mathrm{~F}\right) 5 \mathrm{~d}^{3}{ }^{10} \mathrm{H}$ & 28.1 & $4 \mathrm{f}^{8}\left({ }^{7} \mathrm{~F}\right) 5 \mathrm{~d}^{3}{ }^{10} \mathrm{I}$ & 1.400 & & \\
\hline & 23864 & & 7.5 & $4 \mathrm{f}^{9}\left({ }^{6} \mathrm{H}\right) 6 \mathrm{~s} 6 \mathrm{p}{ }^{8} \mathrm{I}$ & 6.6 & $4 \mathrm{f}^{8}\left({ }^{7} \mathrm{~F}\right) 5 \mathrm{~d}^{2} 6 \mathrm{~s}^{8} \mathrm{I}$ & 1.309 & & \\
\hline & 24221 & & 8.9 & $4 \mathrm{f}^{8}\left({ }^{7} \mathrm{~F}\right) 5 \mathrm{~d}^{2} 6 \mathrm{~s}^{8} \mathrm{~F}$ & 7.2 & $4 \mathrm{f}^{8}\left({ }^{7} \mathrm{~F}\right) 5 \mathrm{~d}^{2} 6 \mathrm{~s}^{8} \mathrm{G}$ & 1.341 & & \\
\hline & 24364 & & 8.1 & $4 f^{8}\left({ }^{7} F\right) 5 d^{2} 6 s^{8} G$ & 7.8 & $4 f^{8}\left({ }^{7} F\right) 5 d^{2} 6 s{ }^{4} G$ & 1.305 & & \\
\hline & 24551 & & 9.6 & $4 f^{8}\left({ }^{7} F\right) 5 d^{2} 6 s^{8} H$ & 8.7 & $4 f^{8}\left({ }^{7} \mathrm{~F}\right) 5 \mathrm{~d}^{2} 6 \mathrm{~s}^{8} \mathrm{H}$ & 1.408 & & \\
\hline & 24579 & & 13.2 & $4 f^{8}\left({ }^{7} F\right) 5 d^{2} 6 s{ }^{4} G$ & 13.1 & $4 \mathrm{f}^{8}\left({ }^{7} \mathrm{~F}\right) 5 \mathrm{~d}^{2} 6 \mathrm{~s}^{8} \mathrm{~F}$ & 1.405 & & \\
\hline & 24641 & & 12.3 & $4 \mathrm{f}^{9}\left({ }^{6} \mathrm{~F}\right) 6 \mathrm{~s} 6 \mathrm{p}{ }^{8} \mathrm{G}$ & 7.1 & $4 \mathrm{f}^{9}\left({ }^{6} \mathrm{~F}\right) 6 \mathrm{~s} 6 \mathrm{p}{ }^{6} \mathrm{G}$ & 1.309 & & \\
\hline
\end{tabular}


Table 1. Continued.

\begin{tabular}{|c|c|c|c|c|c|c|c|c|c|}
\hline$E_{\exp }$ & $E_{\text {calc }}$ & $\Delta E$ & $\%$ & Main comp. & $\%$ & Sec. comp. & $g_{J \text { calc }}$ & $g_{J} \exp$ & $\Delta g_{J}$ \\
\hline & 24670 & & 7.1 & $4 \mathrm{f}^{8}\left({ }^{5} \mathrm{G}\right) 5 \mathrm{~d} 6 \mathrm{~s}^{2}{ }^{6} \mathrm{~F}$ & 6.9 & $4 \mathrm{f}^{8}\left({ }^{5} \mathrm{G}\right) 5 \mathrm{~d} 6 \mathrm{~s}^{2}{ }^{6} \mathrm{~F}$ & 1.320 & & \\
\hline & 25012 & & 42.7 & $4 \mathrm{f}^{8}\left({ }^{7} \mathrm{~F}\right) 5 \mathrm{~d}^{3}{ }^{10} \mathrm{P}$ & 26.4 & $4 f^{8}\left({ }^{7} F\right) 5 d^{3}{ }^{10} D$ & 1.698 & & \\
\hline & 25074 & & 11.2 & $4 \mathrm{f}^{8}\left({ }^{7} \mathrm{~F}\right) 5 \mathrm{~d}^{2} 6 \mathrm{~s}{ }^{6} \mathrm{I}$ & 6.7 & $4 f^{8}\left({ }^{7} F\right) 5 d^{2} 6 s^{8} F$ & 1.289 & & \\
\hline & 25160 & & 11.6 & $4 f^{9}\left({ }^{6} F\right) 6 s 6 p{ }^{4} G$ & 6.9 & $4 f^{8}\left({ }^{7} F\right) 5 d^{2} 6 s{ }^{4} I$ & 1.268 & & \\
\hline & 25233 & & 16.2 & $4 f^{9}\left({ }^{6} F\right) 6 s 6 p{ }^{4} G$ & 12.3 & $4 \mathrm{f}^{9}\left({ }^{6} \mathrm{~F}\right) 6 \mathrm{~s} 6 \mathrm{p}{ }^{6} \mathrm{~F}$ & 1.277 & & \\
\hline & 25303 & & 12.5 & $4 \mathrm{f}^{8}\left({ }^{7} \mathrm{~F}\right) 5 \mathrm{~d}^{2} 6 \mathrm{~s}^{8} \mathrm{D}$ & 7.2 & $4 f^{9}\left({ }^{6} \mathrm{H}\right) 6 \mathrm{~s} 6 \mathrm{p}{ }^{6} \mathrm{G}$ & 1.397 & & \\
\hline & 25381 & & 30.3 & $4 \mathrm{f}^{8}\left({ }^{7} \mathrm{~F}\right) 5 \mathrm{~d}^{3}{ }^{10} \mathrm{I}$ & 9.6 & $4 f^{8}\left({ }^{7} F\right) 5 d^{3}{ }^{10} G$ & 1.367 & & \\
\hline & 25413 & & 18.1 & $4 \mathrm{f}^{9}\left({ }^{6} \mathrm{H}\right) 6 \mathrm{~s} 6 \mathrm{p}{ }^{6} \mathrm{G}$ & 12.2 & $4 \mathrm{f}^{8}\left({ }^{7} \mathrm{~F}\right) 5 \mathrm{~d}^{3}{ }^{10} \mathrm{I}$ & 1.298 & & \\
\hline & 25579 & & 23.9 & $4 f^{8}\left({ }^{7} F\right) 5 d^{2} 6 s{ }^{4} I$ & 8.3 & $4 f^{8}\left({ }^{7} F\right) 5 d^{2} 6 s^{8} D$ & 1.229 & & \\
\hline \multirow[t]{6}{*}{25637.87} & 25634 & 4 & 8.9 & $4 \mathrm{f}^{8}\left({ }^{7} \mathrm{~F}\right) 5 \mathrm{~d}^{2} 6 \mathrm{~s}^{8} \mathrm{I}$ & 7.3 & $4 \mathrm{f}^{8}\left({ }^{7} \mathrm{~F}\right) 5 \mathrm{~d}^{2} 6 \mathrm{~s}^{8} \mathrm{H}$ & 1.348 & 1.334 & -0.014 \\
\hline & 25797 & & 37.8 & $4 \mathrm{f}^{8}\left({ }^{5} \mathrm{~L}\right) 5 \mathrm{~d} 6 \mathrm{~s}^{2}{ }^{6} \mathrm{~L}$ & 4.7 & $4 \mathrm{f}^{8}\left({ }^{7} \mathrm{~F}\right) 5 \mathrm{~d}^{2} 6 \mathrm{~s}^{8} \mathrm{~F}$ & 0.928 & & \\
\hline & 25842 & & 13.6 & $4 \mathrm{f}^{8}\left({ }^{7} \mathrm{~F}\right) 5 \mathrm{~d}^{2} 6 \mathrm{~s}^{8} \mathrm{~F}$ & 11.5 & $4 \mathrm{f}^{8}\left({ }^{5} \mathrm{~L}\right) 5 \mathrm{~d} 6 \mathrm{~s}^{2}{ }^{6} \mathrm{~L}$ & 1.215 & & \\
\hline & 26109 & & 8.9 & $4 f^{8}\left({ }^{7} F\right) 5 d^{2} 6 s^{8} G$ & 7.6 & $4 \mathrm{f}^{8}\left({ }^{7} \mathrm{~F}\right) 5 \mathrm{~d}^{2} 6 \mathrm{~s}{ }^{6} \mathrm{I}$ & 1.342 & & \\
\hline & 26239 & & 9.6 & $4 \mathrm{f}^{9}\left({ }^{6} \mathrm{H}\right) 6 \mathrm{~s} 6 \mathrm{p}{ }^{6} \mathrm{I}$ & 7.4 & $4 \mathrm{f}^{9}\left({ }^{6} \mathrm{H}\right) 6 \mathrm{~s} 6 \mathrm{p}{ }^{4} \mathrm{I}$ & 1.191 & & \\
\hline & 26308 & & 8.6 & $4 \mathrm{f}^{8}\left({ }^{7} \mathrm{~F}\right) 5 \mathrm{~d}^{2} 6 \mathrm{~s}{ }^{6} \mathrm{H}$ & 8.3 & $4 \mathrm{f}^{9}\left({ }^{6} \mathrm{H}\right) 6 \mathrm{~s} 6 \mathrm{p}{ }^{6} \mathrm{I}$ & 1.175 & & \\
\hline \multirow[t]{2}{*}{26553.26} & 26506 & 47 & 10.9 & $4 \mathrm{f}^{9}\left({ }^{6} \mathrm{~F}\right) 6 \mathrm{~s} 6 \mathrm{p}{ }^{6} \mathrm{~F}$ & 10.1 & $4 \mathrm{f}^{8}\left({ }^{5} \mathrm{D}\right) 5 \mathrm{~d} 6 \mathrm{~s}^{2}{ }^{6} \mathrm{G}$ & 1.334 & & \\
\hline & 26602 & & 11.6 & $4 \mathrm{f}^{8}\left({ }^{7} \mathrm{~F}\right) 5 \mathrm{~d}^{2} 6 \mathrm{~s}^{8} \mathrm{I}$ & 10.5 & $4 \mathrm{f}^{9}\left({ }^{6} \mathrm{~F}\right) 6 \mathrm{~s} 6 \mathrm{p}{ }^{6} \mathrm{~F}$ & 1.266 & & \\
\hline \multicolumn{10}{|l|}{$J=13 / 2$} \\
\hline 285.500 & 286 & -1 & 59.2 & $4 \mathrm{f}^{8}\left({ }^{7} \mathrm{~F}\right) 5 \mathrm{~d} 6 \mathrm{~s}^{2}{ }^{8} \mathrm{G}$ & 25.1 & $4 \mathrm{f}^{8}\left({ }^{7} \mathrm{~F}\right) 5 \mathrm{~d} 6 \mathrm{~s}^{2}{ }^{8} \mathrm{~F}$ & 1.466 & 1.464 & -0.002 \\
\hline 3719.705 & 3668 & 52 & 65.0 & $4 \mathrm{f}^{8}\left({ }^{7} \mathrm{~F}\right) 5 \mathrm{~d} 6 \mathrm{~s}^{2}{ }^{8} \mathrm{~F}$ & 22.2 & $4 \mathrm{f}^{8}\left({ }^{7} \mathrm{~F}\right) 5 \mathrm{~d} 6 \mathrm{~s}^{2}{ }^{8} \mathrm{G}$ & 1.504 & 1.505 & 0.001 \\
\hline 6351.750 & 6306 & 46 & 67.8 & $4 \mathrm{f}^{8}\left({ }^{7} \mathrm{~F}\right) 5 \mathrm{~d} 6 \mathrm{~s}^{2}{ }^{8} \mathrm{H}$ & 11.1 & $4 \mathrm{f}^{8}\left({ }^{7} \mathrm{~F}\right) 5 \mathrm{~d} 6 \mathrm{~s}^{2}{ }^{6} \mathrm{G}$ & 1.355 & 1.350 & -0.005 \\
\hline 7059.900 & 7039 & 21 & 63.2 & $4 \mathrm{f}^{8}\left({ }^{7} \mathrm{~F}\right) 5 \mathrm{~d} 6 \mathrm{~s}^{2}{ }^{6} \mathrm{G}$ & 11.4 & $4 \mathrm{f}^{8}\left({ }^{7} \mathrm{~F}\right) 5 \mathrm{~d} 6 \mathrm{~s}^{2}{ }^{6} \mathrm{H}$ & 1.370 & 1.380 & 0.010 \\
\hline 8277.040 & 8266 & 11 & 56.4 & $4 \mathrm{f}^{8}\left({ }^{7} \mathrm{~F}\right) 5 \mathrm{~d}^{2} 6 \mathrm{~s}{ }^{10} \mathrm{G}$ & 19.3 & $4 \mathrm{f}^{8}\left({ }^{7} \mathrm{~F}\right) 5 \mathrm{~d}^{2} 6 \mathrm{~s}{ }^{10} \mathrm{~F}$ & 1.555 & 1.570 & 0.015 \\
\hline 9763.020 & 9757 & 6 & 70.4 & $4 \mathrm{f}^{8}\left({ }^{7} \mathrm{~F}\right) 5 \mathrm{~d} 6 \mathrm{~s}^{2}{ }^{6} \mathrm{H}$ & 12.7 & $4 \mathrm{f}^{8}\left({ }^{7} \mathrm{~F}\right) 5 \mathrm{~d} 6 \mathrm{~s}^{2}{ }^{8} \mathrm{H}$ & 1.297 & 1.300 & 0.003 \\
\hline 11425.94 & 11417 & 9 & 31.5 & $4 f^{8}\left({ }^{7} F\right) 5 d^{2} 6 s{ }^{10} F$ & 12.0 & $4 \mathrm{f}^{8}\left({ }^{7} \mathrm{~F}\right) 5 \mathrm{~d}^{2} 6 \mathrm{~s}{ }^{10} \mathrm{D}$ & 1.569 & & \\
\hline 12475.74 & 12465 & 11 & 25.0 & $4 f^{8}\left({ }^{7} F\right) 5 d^{2} 6 s^{8} G$ & 15.8 & $4 \mathrm{f}^{8}\left({ }^{7} \mathrm{~F}\right) 5 \mathrm{~d}^{2} 6 \mathrm{~s}{ }^{10} \mathrm{D}$ & 1.503 & & \\
\hline 12906.60 & 12858 & 48 & 42.3 & $4 \mathrm{f}^{8}\left({ }^{7} \mathrm{~F}\right) 5 \mathrm{~d}^{2} 6 \mathrm{~s}{ }^{10} \mathrm{D}$ & 22.6 & $4 \mathrm{f}^{8}\left({ }^{7} \mathrm{~F}\right) 5 \mathrm{~d}^{2} 6 \mathrm{~s}{ }^{10} \mathrm{H}$ & 1.544 & & \\
\hline \multirow[t]{7}{*}{13116.48} & 13141 & -25 & 24.8 & $4 \mathrm{f}^{8}\left({ }^{7} \mathrm{~F}\right) 5 \mathrm{~d}^{2} 6 \mathrm{~s}{ }^{10} \mathrm{D}$ & 17.9 & $4 \mathrm{f}^{8}\left({ }^{7} \mathrm{~F}\right) 5 \mathrm{~d}^{2} 6 \mathrm{~s}{ }^{10} \mathrm{I}$ & 1.525 & & \\
\hline & 15013 & & 47.2 & $4 \mathrm{f}^{8}\left({ }^{7} \mathrm{~F}\right) 5 \mathrm{~d}^{2} 6 \mathrm{~s}{ }^{10} \mathrm{I}$ & 27.0 & $4 \mathrm{f}^{8}\left({ }^{7} \mathrm{~F}\right) 5 \mathrm{~d}^{2} 6 \mathrm{~s}{ }^{10} \mathrm{H}$ & 1.383 & & \\
\hline & 15193 & & 22.9 & $4 \mathrm{f}^{8}\left({ }^{7} \mathrm{~F}\right) 5 \mathrm{~d}^{2} 6 \mathrm{~s}^{8} \mathrm{~F}$ & 17.0 & $4 \mathrm{f}^{8}\left({ }^{7} \mathrm{~F}\right) 5 \mathrm{~d}^{2} 6 \mathrm{~s}{ }^{10} \mathrm{D}$ & 1.489 & & \\
\hline & 15663 & & 42.1 & $4 \mathrm{f}^{8}\left({ }^{7} \mathrm{~F}\right) 5 \mathrm{~d}^{2} 6 \mathrm{~s}{ }^{10} \mathrm{D}$ & 14.6 & $4 \mathrm{f}^{8}\left({ }^{7} \mathrm{~F}\right) 5 \mathrm{~d}^{2} 6 \mathrm{~s}^{8} \mathrm{H}$ & 1.521 & & \\
\hline & 16322 & & 14.8 & $4 \mathrm{f}^{9}\left({ }^{6} \mathrm{H}\right) 6 \mathrm{~s} 6 \mathrm{p}{ }^{4} \mathrm{H}$ & 13.9 & $4 f^{9}\left({ }^{6} \mathrm{H}\right) 6 \mathrm{~s} 6 \mathrm{p}{ }^{6} \mathrm{G}$ & 1.370 & & \\
\hline & 16397 & & 14.4 & $4 \mathrm{f}^{8}\left({ }^{7} \mathrm{~F}\right) 5 \mathrm{~d}^{2} 6 \mathrm{~s}^{8} \mathrm{~F}$ & 8.8 & $4 \mathrm{f}^{8}\left({ }^{7} \mathrm{~F}\right) 5 \mathrm{~d}^{2} 6 \mathrm{~s}^{8} \mathrm{I}$ & 1.409 & & \\
\hline & 17262 & & 64.2 & $4 f^{8}\left({ }^{7} F\right) 5 d^{2} 6 s{ }^{10} G$ & 9.9 & $4 \mathrm{f}^{8}\left({ }^{7} \mathrm{~F}\right) 5 \mathrm{~d}^{2} 6 \mathrm{~s}{ }^{10} \mathrm{G}$ & 1.530 & & \\
\hline \multirow[t]{11}{*}{17875.98} & 17886 & -10 & 32.6 & $4 f^{8}\left({ }^{7} F\right) 5 d^{2} 6 s^{8} I$ & 18.5 & $4 f^{8}\left({ }^{7} F\right) 5 d^{2} 6 s^{8} H$ & 1.288 & & \\
\hline & 17951 & & 24.6 & $4 \mathrm{f}^{8}\left({ }^{7} \mathrm{~F}\right) 5 \mathrm{~d}^{2} 6 \mathrm{~s}{ }^{6} \mathrm{H}$ & 11.2 & $4 f^{8}\left({ }^{7} F\right) 5 d^{2} 6 s{ }^{6} G$ & 1.314 & & \\
\hline & 18434 & & 31.1 & $4 \mathrm{f}^{9}\left({ }^{6} \mathrm{H}\right) 6 \mathrm{~s} 6 \mathrm{p}{ }^{4} \mathrm{H}$ & 26.1 & $4 \mathrm{f}^{9}\left({ }^{6} \mathrm{H}\right) 6 \mathrm{~s} 6 \mathrm{p}{ }^{6} \mathrm{G}$ & 1.315 & & \\
\hline & 18867 & & 25.7 & $4 f^{9}\left({ }^{6} H\right) 6 s 6 p{ }^{6} G$ & 20.7 & $4 \mathrm{f}^{9}\left({ }^{6} \mathrm{H}\right) 6 \mathrm{~s} 6 \mathrm{p}^{8} \mathrm{H}$ & 1.343 & & \\
\hline & 19156 & & 18.4 & $4 \mathrm{f}^{8}\left({ }^{7} \mathrm{~F}\right) 5 \mathrm{~d}^{2} 6 \mathrm{~s}^{8} \mathrm{G}$ & 14.7 & $4 f^{8}\left({ }^{7} F\right) 5 d^{2} 6 s^{8} G$ & 1.420 & & \\
\hline & 19555 & & 21.7 & $4 \mathrm{f}^{8}\left({ }^{7} \mathrm{~F}\right) 5 \mathrm{~d}^{2} 6 \mathrm{~s}{ }^{6} \mathrm{G}$ & 6.8 & $4 f^{8}\left({ }^{7} F\right) 5 d^{2} 6 s^{8} F$ & 1.371 & & \\
\hline & 19666 & & 32.0 & $4 \mathrm{f}^{8}\left({ }^{7} \mathrm{~F}\right) 5 \mathrm{~d}^{3}{ }^{10} \mathrm{~F}$ & 22.7 & $4 \mathrm{f}^{8}\left({ }^{7} \mathrm{~F}\right) 5 \mathrm{~d}^{3}{ }^{10} \mathrm{D}$ & 1.568 & & \\
\hline & 19809 & & 8.3 & $4 \mathrm{f}^{9}\left({ }^{6} \mathrm{H}\right) 6 \mathrm{~s} 6 \mathrm{p}{ }^{8} \mathrm{G}$ & 7.9 & $4 \mathrm{f}^{9}\left({ }^{6} \mathrm{H}\right) 6 \mathrm{~s} 6 \mathrm{p}{ }^{6} \mathrm{I}$ & 1.390 & & \\
\hline & 19831 & & 37.2 & $4 f^{8}\left({ }^{7} F\right) 5 d^{2} 6 s^{8} G$ & 8.2 & $4 f^{8}\left({ }^{7} F\right) 5 d^{2} 6 s^{8} G$ & 1.444 & & \\
\hline & 20113 & & 14.5 & $4 \mathrm{f}^{9}\left({ }^{6} \mathrm{H}\right) 6 \mathrm{~s} 6 \mathrm{p}{ }^{6} \mathrm{I}$ & 8.4 & $4 \mathrm{f}^{9}\left({ }^{6} \mathrm{H}\right) 6 \mathrm{~s} 6 \mathrm{p}{ }^{4} \mathrm{I}$ & 1.311 & & \\
\hline & 20379 & & 27.2 & $4 \mathrm{f}^{8}\left({ }^{7} \mathrm{~F}\right) 5 \mathrm{~d}^{2} 6 \mathrm{~s}{ }^{6} \mathrm{I}$ & 11.8 & $4 f^{8}\left({ }^{7} F\right) 5 d^{2} 6 s{ }^{6} I$ & 1.239 & & \\
\hline
\end{tabular}


Table 1. Continued.

\begin{tabular}{|c|c|c|c|c|c|c|c|c|c|}
\hline$E_{\exp }$ & $E_{\text {calc }}$ & $\Delta E$ & $\%$ & Main comp. & $\%$ & Sec. comp. & $g_{J \text { calc }}$ & $g_{J \exp }$ & $\Delta g_{J}$ \\
\hline & 20858 & & 23.0 & $4 f^{8}\left({ }^{7} F\right) 5 d^{2} 6 s^{8} F$ & 15.5 & $4 f^{8}\left({ }^{7} \mathrm{~F}\right) 5 \mathrm{~d}^{2} 6 \mathrm{~s}{ }^{10} \mathrm{~F}$ & 1.465 & & \\
\hline & 21088 & & 17.5 & $4 \mathrm{f}^{9}\left({ }^{6} \mathrm{H}\right) 6 \mathrm{~s} 6 \mathrm{p}{ }^{6} \mathrm{G}$ & 10.2 & $4 \mathrm{f}^{8}\left({ }^{5} \mathrm{D}\right) 5 \mathrm{~d} 6 \mathrm{~s}^{2}{ }^{6} \mathrm{G}$ & 1.376 & & \\
\hline & 21163 & & 18.4 & $4 f^{8}\left({ }^{7} \mathrm{~F}\right) 5 \mathrm{~d}^{2} 6 \mathrm{~s}{ }^{10} \mathrm{~F}$ & 6.0 & $4 f^{9}\left({ }^{6} \mathrm{H}\right) 6 \mathrm{~s} 6 \mathrm{p}{ }^{6} \mathrm{G}$ & 1.430 & & \\
\hline & 21358 & & 26.8 & $4 \mathrm{f}^{8}\left({ }^{7} \mathrm{~F}\right) 5 \mathrm{~d}^{2} 6 \mathrm{~s}{ }^{4} \mathrm{H}$ & 16.2 & $4 \mathrm{f}^{8}\left({ }^{7} \mathrm{~F}\right) 5 \mathrm{~d}^{2} 6 \mathrm{~s}{ }^{6} \mathrm{H}$ & 1.296 & & \\
\hline & 21425 & & 11.4 & $4 \mathrm{f}^{9}\left({ }^{6} \mathrm{H}\right) 6 \mathrm{~s} 6 \mathrm{p}{ }^{6} \mathrm{H}$ & 11.2 & $4 f^{9}\left({ }^{6} \mathrm{H}\right) 6 \mathrm{~s} 6 \mathrm{p}{ }^{8} \mathrm{I}$ & 1.310 & & \\
\hline & 21610 & & 11.1 & $4 \mathrm{f}^{9}\left({ }^{6} \mathrm{H}\right) 6 \mathrm{~s} 6 \mathrm{p}{ }^{6} \mathrm{H}$ & 8.6 & $4 \mathrm{f}^{9}\left({ }^{6} \mathrm{H}\right) 6 \mathrm{~s} 6 \mathrm{p}{ }^{8} \mathrm{G}$ & 1.312 & & \\
\hline & 21691 & & 15.6 & $4 \mathrm{f}^{8}\left({ }^{7} \mathrm{~F}\right) 5 \mathrm{~d}^{2} 6 \mathrm{~s}{ }^{10} \mathrm{~F}$ & 9.4 & $4 \mathrm{f}^{8}\left({ }^{7} \mathrm{~F}\right) 5 \mathrm{~d}^{2} 6 \mathrm{~s}{ }^{6} \mathrm{G}$ & 1.393 & & \\
\hline & 21713 & & 9.4 & $4 \mathrm{f}^{8}\left({ }^{7} \mathrm{~F}\right) 5 \mathrm{~d}^{2} 6 \mathrm{~s}^{8} \mathrm{G}$ & 9.1 & $4 \mathrm{f}^{9}\left({ }^{6} \mathrm{H}\right) 6 \mathrm{~s} 6 \mathrm{p}{ }^{6} \mathrm{H}$ & 1.329 & & \\
\hline & 22100 & & 17.9 & $4 \mathrm{f}^{8}\left({ }^{7} \mathrm{~F}\right) 5 \mathrm{~d}^{2} 6 \mathrm{~s}{ }^{6} \mathrm{H}$ & 8.6 & $4 f^{8}\left({ }^{7} F\right) 5 d^{2} 6 s{ }^{6} G$ & 1.290 & & \\
\hline & 22438 & & 23.2 & $4 \mathrm{f}^{8}\left({ }^{7} \mathrm{~F}\right) 5 \mathrm{~d}^{3}{ }^{10} \mathrm{D}$ & 7.0 & $4 \mathrm{f}^{8}\left({ }^{7} \mathrm{~F}\right) 5 \mathrm{~d}^{2} 6 \mathrm{~s}^{8} \mathrm{~F}$ & 1.513 & & \\
\hline & 22620 & & 30.6 & $4 \mathrm{f}^{8}\left({ }^{7} \mathrm{~F}\right) 5 \mathrm{~d}^{3}{ }^{10} \mathrm{D}$ & 7.9 & $4 f^{8}\left({ }^{7} \mathrm{~F}\right) 5 \mathrm{~d}^{3}{ }^{10} \mathrm{~F}$ & 1.548 & & \\
\hline & 22733 & & 13.6 & $4 \mathrm{f}^{8}\left({ }^{5} \mathrm{D}\right) 5 \mathrm{~d} 6 \mathrm{~s}^{2}{ }^{6} \mathrm{G}$ & 13.3 & $4 f^{9}\left({ }^{6} \mathrm{H}\right) 6 \mathrm{~s} 6 \mathrm{p}{ }^{6} \mathrm{G}$ & 1.346 & & \\
\hline 23043.43 & 23030 & 14 & 9.9 & $4 \mathrm{f}^{8}\left({ }^{7} \mathrm{~F}\right) 5 \mathrm{~d}^{2} 6 \mathrm{~s}{ }^{6} \mathrm{H}$ & 9.2 & $4 \mathrm{f}^{8}\left({ }^{7} \mathrm{~F}\right) 5 \mathrm{~d}^{2} 6 \mathrm{~s}{ }^{6} \mathrm{G}$ & 1.363 & 1.391 & 0.028 \\
\hline \multirow[t]{13}{*}{23112.35} & 23095 & 17 & 10.5 & $4 f^{8}\left({ }^{7} F\right) 5 d^{2} 6 s^{8} I$ & 7.4 & $4 \mathrm{f}^{8}\left({ }^{7} \mathrm{~F}\right) 5 \mathrm{~d}^{2} 6 \mathrm{~s}^{8} \mathrm{~F}$ & 1.368 & & \\
\hline & 23235 & & 34.4 & $4 \mathrm{f}^{8}\left({ }^{7} \mathrm{~F}\right) 5 \mathrm{~d}^{3}{ }^{10} \mathrm{H}$ & 22.9 & $4 \mathrm{f}^{8}\left({ }^{7} \mathrm{~F}\right) 5 \mathrm{~d}^{3}{ }^{10} \mathrm{I}$ & 1.448 & & \\
\hline & 23262 & & 14.6 & $4 f^{8}\left({ }^{7} F\right) 5 d^{2} 6 s^{8} F$ & 8.1 & $4 f^{8}\left({ }^{7} F\right) 5 d^{2} 6 s^{8} G$ & 1.412 & & \\
\hline & 23308 & & 12.3 & $4 f^{9}\left({ }^{6} \mathrm{H}\right) 6 \mathrm{~s} 6 \mathrm{p}{ }^{4} \mathrm{I}$ & 12.1 & $4 \mathrm{f}^{9}\left({ }^{6} \mathrm{H}\right) 6 \mathrm{~s} 6 \mathrm{p}{ }^{8} \mathrm{H}$ & 1.286 & & \\
\hline & 23515 & & 8.8 & $4 f^{9}\left({ }^{6} \mathrm{H}\right) 6 \mathrm{~s} 6 \mathrm{p}{ }^{6} \mathrm{G}$ & 5.5 & $4 \mathrm{f}^{8}\left({ }^{5} \mathrm{G}\right) 5 \mathrm{~d} 6 \mathrm{~s}^{2}{ }^{6} \mathrm{H}$ & 1.309 & & \\
\hline & 23872 & & 15.5 & $4 \mathrm{f}^{9}\left({ }^{6} \mathrm{~F}\right) 6 \mathrm{~s} 6 \mathrm{p}{ }^{8} \mathrm{~F}$ & 13.2 & $4 f^{9}\left({ }^{6} F\right) 6 s 6 p{ }^{8} G$ & 1.420 & & \\
\hline & 23940 & & 16.5 & $4 \mathrm{f}^{8}\left({ }^{7} \mathrm{~F}\right) 5 \mathrm{~d}^{2} 6 \mathrm{~s}^{8} \mathrm{H}$ & 10.2 & $4 \mathrm{f}^{8}\left({ }^{7} \mathrm{~F}\right) 5 \mathrm{~d}^{2} 6 \mathrm{~s}^{8} \mathrm{H}$ & 1.360 & & \\
\hline & 24060 & & 16.8 & $4 \mathrm{f}^{8}\left({ }^{7} \mathrm{~F}\right) 5 \mathrm{~d}^{2} 6 \mathrm{~s}^{4} \mathrm{I}$ & 9.1 & $4 f^{9}\left({ }^{6} \mathrm{~F}\right) 6 \mathrm{~s} 6 \mathrm{p}{ }^{8} \mathrm{~F}$ & 1.333 & & \\
\hline & 24379 & & 15.9 & $4 \mathrm{f}^{8}\left({ }^{7} \mathrm{~F}\right) 5 \mathrm{~d}^{2} 6 \mathrm{~s}{ }^{6} \mathrm{I}$ & 13.2 & $4 \mathrm{f}^{8}\left({ }^{7} \mathrm{~F}\right) 5 \mathrm{~d}^{2} 6 \mathrm{~s}{ }^{6} \mathrm{I}$ & 1.270 & & \\
\hline & 24548 & & 21.1 & $4 \mathrm{f}^{8}\left({ }^{7} \mathrm{~F}\right) 5 \mathrm{~d}^{2} 6 \mathrm{~s}^{4} \mathrm{I}$ & 6.8 & $4 f^{8}\left({ }^{7} F\right) 5 d^{2} 6 s{ }^{6} G$ & 1.282 & & \\
\hline & 24868 & & 24.6 & $4 \mathrm{f}^{9}\left({ }^{6} \mathrm{H}\right) 6 \mathrm{~s} 6 \mathrm{p}{ }^{6} \mathrm{I}$ & 17.6 & $4 \mathrm{f}^{9}\left({ }^{6} \mathrm{H}\right) 6 \mathrm{~s} 6 \mathrm{p}{ }^{8} \mathrm{I}$ & 1.212 & & \\
\hline & 24935 & & 51.0 & $4 \mathrm{f}^{8}\left({ }^{7} \mathrm{~F}\right) 5 \mathrm{~d}^{3}{ }^{10} \mathrm{I}$ & 19.1 & $4 f^{8}\left({ }^{7} F\right) 5 d^{3}{ }^{10} G$ & 1.407 & & \\
\hline & 25125 & & 13.2 & $4 \mathrm{f}^{8}\left({ }^{7} \mathrm{~F}\right) 5 \mathrm{~d}^{2} 6 \mathrm{~s}^{8} \mathrm{H}$ & 9.2 & $4 \mathrm{f}^{8}\left({ }^{7} \mathrm{~F}\right) 5 \mathrm{~d}^{2} 6 \mathrm{~s}{ }^{8} \mathrm{H}$ & 1.329 & & \\
\hline \multirow[t]{2}{*}{25373.85} & 25286 & 88 & 11.9 & $4 f^{8}\left({ }^{7} F\right) 5 d^{2} 6 s^{8} I$ & 11.6 & $4 f^{8}\left({ }^{7} \mathrm{~F}\right) 5 \mathrm{~d}^{2} 6 \mathrm{~s}^{8} \mathrm{I}$ & 1.292 & 1.354 & 0.062 \\
\hline & 25526 & & 21.0 & $4 \mathrm{f}^{8}\left({ }^{7} \mathrm{~F}\right) 5 \mathrm{~d}^{2} 6 \mathrm{~s}^{8} \mathrm{~F}$ & 10.4 & $4 f^{8}\left({ }^{7} F\right) 5 d^{2} 6 s^{8} G$ & 1.453 & & \\
\hline 25553.46 & 25560 & -7 & 34.4 & $4 \mathrm{f}^{9}\left({ }^{6} \mathrm{H}\right) 6 \mathrm{~s} 6 \mathrm{p}{ }^{6} \mathrm{H}$ & 7.0 & $4 \mathrm{f}^{9}\left({ }^{6} \mathrm{H}\right) 5 \mathrm{~d} 6 \mathrm{p}{ }^{6} \mathrm{H}$ & 1.297 & 1.328 & 0.031 \\
\hline \multirow[t]{6}{*}{25717.68} & 25749 & -31 & 17.2 & $4 \mathrm{f}^{8}\left({ }^{5} \mathrm{~L}\right) 5 \mathrm{~d} 6 \mathrm{~s}^{2}{ }^{6} \mathrm{~L}$ & 15.4 & $4 f^{9}\left({ }^{6} \mathrm{~F}\right) 6 \mathrm{~s} 6 \mathrm{p}{ }^{8} \mathrm{~F}$ & 1.239 & 1.300 & 0.061 \\
\hline & 25833 & & 33.9 & $4 \mathrm{f}^{8}\left({ }^{5} \mathrm{~L}\right) 5 \mathrm{~d} 6 \mathrm{~s}^{2}{ }^{6} \mathrm{~L}$ & 6.2 & $4 f^{9}\left({ }^{6} F\right) 6 s 6 p{ }^{8} F$ & 1.084 & & \\
\hline & 26079 & & 12.0 & $4 f^{8}\left({ }^{7} F\right) 5 d^{2} 6 s^{8} I$ & 10.3 & $4 f^{9}\left({ }^{6} F\right) 6 s 6 p{ }^{8} F$ & 1.316 & & \\
\hline & 26124 & & 12.8 & $4 f^{8}\left({ }^{7} F\right) 5 d^{2} 6 s^{8} I$ & 8.9 & $4 f^{8}\left({ }^{7} F\right) 5 d^{2} 6 s{ }^{6} G$ & 1.346 & & \\
\hline & 26300 & & 16.2 & $4 \mathrm{f}^{8}\left({ }^{5} \mathrm{~L}\right) 5 \mathrm{~d} 6 \mathrm{~s}^{2}{ }^{6} \mathrm{M}$ & 12.3 & $4 \mathrm{f}^{8}\left({ }^{5} \mathrm{G}\right) 5 \mathrm{~d} 6 \mathrm{~s}^{2}{ }^{6} \mathrm{G}$ & 1.133 & & \\
\hline & 26508 & & 30.5 & $4 \mathrm{f}^{8}\left({ }^{7} \mathrm{~F}\right) 5 \mathrm{~d}^{3}{ }^{10} \mathrm{H}$ & 23.5 & $4 \mathrm{f}^{8}\left({ }^{7} \mathrm{~F}\right) 5 \mathrm{~d}^{3}{ }^{10} \mathrm{G}$ & 1.485 & & \\
\hline \multirow[t]{2}{*}{26592.90} & 26606 & -13 & 36.6 & $4 f^{9}\left({ }^{6} F\right) 6 s 6 p{ }^{6} G$ & 34.7 & $4 f^{9}\left({ }^{6} F\right) 6 s 6 p{ }^{8} G$ & 1.407 & & \\
\hline & 26745 & & 34.8 & $4 \mathrm{f}^{8}\left({ }^{5} \mathrm{~L}\right) 5 \mathrm{~d} 6 \mathrm{~s}^{2}{ }^{6} \mathrm{M}$ & 11.4 & $4 \mathrm{f}^{8}\left({ }^{5} \mathrm{~L}\right) 5 \mathrm{~d} 6 \mathrm{~s}^{2}{ }^{6} \mathrm{~L}$ & 0.901 & & \\
\hline \multicolumn{10}{|l|}{$J=15 / 2$} \\
\hline 462.080 & 551 & -89 & 85.9 & $4 \mathrm{f}^{8}\left({ }^{7} \mathrm{~F}\right) 5 \mathrm{~d} 6 \mathrm{~s}^{2}{ }^{8} \mathrm{G}$ & 4.2 & $4 \mathrm{f}^{8}\left({ }^{7} \mathrm{~F}\right) 5 \mathrm{~d} 6 \mathrm{~s}^{2}{ }^{8} \mathrm{H}$ & 1.457 & 1.456 & -0.001 \\
\hline 5425.060 & 5442 & -17 & 64.9 & $4 \mathrm{f}^{8}\left({ }^{7} \mathrm{~F}\right) 5 \mathrm{~d} 6 \mathrm{~s}^{2}{ }^{8} \mathrm{H}$ & 20.3 & $4 \mathrm{f}^{8}\left({ }^{7} \mathrm{~F}\right) 5 \mathrm{~d} 6 \mathrm{~s}^{2}{ }^{6} \mathrm{H}$ & 1.375 & 1.370 & -0.005 \\
\hline 7767.015 & 7787 & -20 & 67.2 & $4 \mathrm{f}^{8}\left({ }^{7} \mathrm{~F}\right) 5 \mathrm{~d} 6 \mathrm{~s}^{2}{ }^{6} \mathrm{H}$ & 21.7 & $4 \mathrm{f}^{8}\left({ }^{7} \mathrm{~F}\right) 5 \mathrm{~d} 6 \mathrm{~s}^{2}{ }^{8} \mathrm{H}$ & 1.343 & 1.342 & -0.001 \\
\hline 8190.465 & 8192 & -2 & 62.2 & $4 \mathrm{f}^{8}\left({ }^{7} \mathrm{~F}\right) 5 \mathrm{~d}^{2} 6 \mathrm{~s}{ }^{10} \mathrm{G}$ & 13.3 & $4 f^{8}\left({ }^{7} \mathrm{~F}\right) 5 \mathrm{~d}^{2} 6 \mathrm{~s}{ }^{10} \mathrm{~F}$ & 1.532 & 1.540 & 0.008 \\
\hline 11580.68 & 11579 & 1 & 42.7 & $4 \mathrm{f}^{8}\left({ }^{7} \mathrm{~F}\right) 5 \mathrm{~d}^{2} 6 \mathrm{~s}{ }^{10} \mathrm{~F}$ & 16.3 & $4 f^{8}\left({ }^{7} \mathrm{~F}\right) 5 \mathrm{~d}^{2} 6 \mathrm{~s}{ }^{10} \mathrm{H}$ & 1.519 & & \\
\hline 12628.67 & 12636 & -7 & 27.7 & $4 \mathrm{f}^{8}\left({ }^{7} \mathrm{~F}\right) 5 \mathrm{~d}^{2} 6 \mathrm{~s}{ }^{10} \mathrm{H}$ & 27.6 & $4 \mathrm{f}^{8}\left({ }^{7} \mathrm{~F}\right) 5 \mathrm{~d}^{2} 6 \mathrm{~s}{ }^{10} \mathrm{I}$ & 1.455 & & \\
\hline 12932.66 & 12908 & 24 & 31.3 & $4 f^{8}\left({ }^{7} F\right) 5 d^{2} 6 s^{8} G$ & 18.2 & $4 \mathrm{f}^{8}\left({ }^{7} \mathrm{~F}\right) 5 \mathrm{~d}^{2} 6 \mathrm{~s}^{8} \mathrm{G}$ & 1.472 & & \\
\hline
\end{tabular}


Table 1. Continued.

\begin{tabular}{|c|c|c|c|c|c|c|c|c|c|}
\hline$E_{\exp }$ & $E_{\text {calc }}$ & $\Delta E$ & $\%$ & Main comp. & $\%$ & Sec. comp. & $g_{J \text { calc }}$ & $g_{J \exp }$ & $\Delta g_{J}$ \\
\hline 14569.67 & 14602 & -32 & 41.8 & $4 \mathrm{f}^{8}\left({ }^{7} \mathrm{~F}\right) 5 \mathrm{~d}^{2} 6 \mathrm{~s}{ }^{10} \mathrm{I}$ & 30.0 & $4 f^{8}\left({ }^{7} F\right) 5 d^{2} 6 s{ }^{10} H$ & 1.398 & & \\
\hline 14888.11 & 14899 & -11 & 44.1 & $4 \mathrm{f}^{9}\left({ }^{6} \mathrm{H}\right) 6 \mathrm{~s} 6 \mathrm{p}{ }^{8} \mathrm{G}$ & 17.5 & $4 \mathrm{f}^{9}\left({ }^{6} \mathrm{H}\right) 6 \mathrm{~s} 6 \mathrm{p}{ }^{8} \mathrm{H}$ & 1.400 & 1.391 & -0.009 \\
\hline 15387.79 & 15341 & 47 & 31.5 & $4 \mathrm{f}^{8}\left({ }^{7} \mathrm{~F}\right) 5 \mathrm{~d}^{2} 6 \mathrm{~s}^{8} \mathrm{H}$ & 15.2 & $4 \mathrm{f}^{8}\left({ }^{7} \mathrm{~F}\right) 5 \mathrm{~d}^{2} 6 \mathrm{~s}^{8} \mathrm{I}$ & 1.364 & 1.367 & 0.003 \\
\hline 16343.30 & 16346 & -2 & 32.2 & $4 \mathrm{f}^{9}\left({ }^{6} \mathrm{H}\right) 6 \mathrm{~s} 6 \mathrm{p}{ }^{4} \mathrm{I}$ & 19.5 & $4 \mathrm{f}^{9}\left({ }^{6} \mathrm{H}\right) 6 \mathrm{~s} 6 \mathrm{p}{ }^{6} \mathrm{I}$ & 1.274 & 1.397 & 0.123 \\
\hline \multirow[t]{8}{*}{16431.13} & 16474 & -43 & 62.3 & $4 \mathrm{f}^{8}\left({ }^{7} \mathrm{~F}\right) 5 \mathrm{~d}^{2} 6 \mathrm{~s}{ }^{10} \mathrm{G}$ & 12.0 & $4 \mathrm{f}^{8}\left({ }^{7} \mathrm{~F}\right) 5 \mathrm{~d}^{2} 6 \mathrm{~s}{ }^{10} \mathrm{G}$ & 1.515 & 1.460 & -0.055 \\
\hline & 17169 & & 21.9 & $4 \mathrm{f}^{8}\left({ }^{7} \mathrm{~F}\right) 5 \mathrm{~d}^{2} 6 \mathrm{~s}^{8} \mathrm{H}$ & 14.0 & $4 \mathrm{f}^{8}\left({ }^{7} \mathrm{~F}\right) 5 \mathrm{~d}^{2} 6 \mathrm{~s}^{6} \mathrm{I}$ & 1.338 & & \\
\hline & 17763 & & 18.0 & $4 \mathrm{f}^{8}\left({ }^{7} \mathrm{~F}\right) 5 \mathrm{~d}^{2} 6 \mathrm{~s}{ }^{6} \mathrm{H}$ & 17.8 & $4 \mathrm{f}^{9}\left({ }^{6} \mathrm{H}\right) 6 \mathrm{~s} 6 \mathrm{p}{ }^{6} \mathrm{H}$ & 1.324 & & \\
\hline & 18022 & & 16.1 & $4 \mathrm{f}^{9}\left({ }^{6} \mathrm{H}\right) 6 \mathrm{~s} 6 \mathrm{p}{ }^{6} \mathrm{H}$ & 14.0 & $4 \mathrm{f}^{8}\left({ }^{7} \mathrm{~F}\right) 5 \mathrm{~d}^{2} 6 \mathrm{~s}{ }^{6} \mathrm{H}$ & 1.324 & & \\
\hline & 18750 & & 14.5 & $4 f^{8}\left({ }^{7} F\right) 5 d^{2} 6 s^{8} G$ & 14.1 & $4 f^{8}\left({ }^{7} F\right) 5 d^{2} 6 s^{8} G$ & 1.411 & & \\
\hline & 19177 & & 21.6 & $4 \mathrm{f}^{9}\left({ }^{6} \mathrm{H}\right) 6 \mathrm{~s} 6 \mathrm{p}{ }^{8} \mathrm{I}$ & 20.5 & $4 \mathrm{f}^{9}\left({ }^{6} \mathrm{H}\right) 6 \mathrm{~s} 6 \mathrm{p}{ }^{8} \mathrm{H}$ & 1.325 & & \\
\hline & 19381 & & 23.8 & $4 \mathrm{f}^{8}\left({ }^{7} \mathrm{~F}\right) 5 \mathrm{~d}^{2} 6 \mathrm{~s}^{8} \mathrm{G}$ & 22.4 & $4 \mathrm{f}^{8}\left({ }^{7} \mathrm{~F}\right) 5 \mathrm{~d}^{2} 6 \mathrm{~s}^{8} \mathrm{G}$ & 1.426 & & \\
\hline & 19724 & & 15.7 & $4 \mathrm{f}^{8}\left({ }^{7} \mathrm{~F}\right) 5 \mathrm{~d}^{2} 6 \mathrm{~s}^{8} \mathrm{H}$ & 8.2 & $4 \mathrm{f}^{8}\left({ }^{7} \mathrm{~F}\right) 5 \mathrm{~d}^{2} 6 \mathrm{~s}^{8} \mathrm{H}$ & 1.432 & & \\
\hline \multirow[t]{11}{*}{19920.41} & 19917 & 3 & 36.9 & $4 \mathrm{f}^{8}\left({ }^{7} \mathrm{~F}\right) 5 \mathrm{~d}^{3}{ }^{10} \mathrm{~F}$ & 29.5 & $4 \mathrm{f}^{8}\left({ }^{7} \mathrm{~F}\right) 5 \mathrm{~d}^{3}{ }^{10} \mathrm{~F}$ & 1.542 & & \\
\hline & 19949 & & 16.2 & $4 \mathrm{f}^{8}\left({ }^{7} \mathrm{~F}\right) 5 \mathrm{~d}^{2} 6 \mathrm{~s}{ }^{6} \mathrm{I}$ & 12.4 & $4 \mathrm{f}^{8}\left({ }^{7} \mathrm{~F}\right) 5 \mathrm{~d}^{2} 6 \mathrm{~s}{ }^{10} \mathrm{~F}$ & 1.352 & & \\
\hline & 20295 & & 41.5 & $4 \mathrm{f}^{8}\left({ }^{7} \mathrm{~F}\right) 5 \mathrm{~d}^{2} 6 \mathrm{~s}{ }^{10} \mathrm{~F}$ & 9.5 & $4 \mathrm{f}^{8}\left({ }^{7} \mathrm{~F}\right) 5 \mathrm{~d}^{2} 6 \mathrm{~s}{ }^{10} \mathrm{~F}$ & 1.512 & & \\
\hline & 20406 & & 26.2 & $4 \mathrm{f}^{8}\left({ }^{7} \mathrm{~F}\right) 5 \mathrm{~d}^{2} 6 \mathrm{~s}{ }^{6} \mathrm{H}$ & 23.5 & $4 f^{8}\left({ }^{7} F\right) 5 d^{2} 6 s^{4} I$ & 1.306 & & \\
\hline & 21015 & & 29.5 & $4 \mathrm{f}^{9}\left({ }^{6} \mathrm{H}\right) 6 \mathrm{~s} 6 \mathrm{p}{ }^{8} \mathrm{H}$ & 16.0 & $4 \mathrm{f}^{9}\left({ }^{6} \mathrm{H}\right) 6 \mathrm{~s} 6 \mathrm{p}{ }^{6} \mathrm{I}$ & 1.309 & & \\
\hline & 21113 & & 17.3 & $4 \mathrm{f}^{8}\left({ }^{7} \mathrm{~F}\right) 5 \mathrm{~d}^{2} 6 \mathrm{~s}^{8} \mathrm{I}$ & 14.1 & $4 f^{8}\left({ }^{7} F\right) 5 d^{2} 6 s^{8} I$ & 1.356 & & \\
\hline & 21706 & & 33.0 & $4 \mathrm{f}^{9}\left({ }^{6} \mathrm{H}\right) 6 \mathrm{~s} 6 \mathrm{p}{ }^{6} \mathrm{H}$ & 9.0 & $4 \mathrm{f}^{8}\left({ }^{7} \mathrm{~F}\right) 5 \mathrm{~d}^{2} 6 \mathrm{~s}^{8} \mathrm{G}$ & 1.348 & & \\
\hline & 21876 & & 25.8 & $4 \mathrm{f}^{8}\left({ }^{7} \mathrm{~F}\right) 5 \mathrm{~d}^{2} 6 \mathrm{~s}^{8} \mathrm{G}$ & 16.6 & $4 \mathrm{f}^{9}\left({ }^{6} \mathrm{H}\right) 6 \mathrm{~s} 6 \mathrm{p}{ }^{6} \mathrm{H}$ & 1.402 & & \\
\hline & 22493 & & 32.9 & $4 \mathrm{f}^{8}\left({ }^{7} \mathrm{~F}\right) 5 \mathrm{~d}^{3}{ }^{10} \mathrm{H}$ & 19.9 & $4 \mathrm{f}^{8}\left({ }^{7} \mathrm{~F}\right) 5 \mathrm{~d}^{3}{ }^{10} \mathrm{I}$ & 1.398 & & \\
\hline & 22535 & & 27.1 & $4 f^{8}\left({ }^{7} F\right) 5 d^{2} 6 s{ }^{4} I$ & 13.0 & $4 \mathrm{f}^{8}\left({ }^{7} \mathrm{~F}\right) 5 \mathrm{~d}^{3}{ }^{10} \mathrm{H}$ & 1.327 & & \\
\hline & 22767 & & 24.2 & $4 \mathrm{f}^{8}\left({ }^{7} \mathrm{~F}\right) 5 \mathrm{~d}^{2} 6 \mathrm{~s}{ }^{6} \mathrm{H}$ & 16.7 & $4 \mathrm{f}^{8}\left({ }^{7} \mathrm{~F}\right) 5 \mathrm{~d}^{2} 6 \mathrm{~s}{ }^{6} \mathrm{H}$ & 1.316 & & \\
\hline 23031.84 & 23010 & 22 & 24.7 & $4 \mathrm{f}^{9}\left({ }^{6} \mathrm{H}\right) 6 \mathrm{~s} 6 \mathrm{p}^{8} \mathrm{I}$ & 23.9 & $4 \mathrm{f}^{9}\left({ }^{6} \mathrm{H}\right) 6 \mathrm{~s} 6 \mathrm{p}{ }^{6} \mathrm{I}$ & 1.301 & 1.240 & -0.061 \\
\hline \multirow[t]{9}{*}{23147.92} & 23220 & -72 & 21.1 & $4 f^{8}\left({ }^{7} F\right) 5 d^{2} 6 s^{8} G$ & 18.1 & $4 f^{8}\left({ }^{7} \mathrm{~F}\right) 5 \mathrm{~d}^{2} 6 \mathrm{~s}^{8} \mathrm{H}$ & 1.371 & 1.339 & -0.032 \\
\hline & 23259 & & 19.3 & $4 \mathrm{f}^{8}\left({ }^{7} \mathrm{~F}\right) 5 \mathrm{~d}^{2} 6 \mathrm{~s}^{8} \mathrm{H}$ & 10.0 & $4 f^{8}\left({ }^{7} F\right) 5 d^{2} 6 s^{8} I$ & 1.327 & & \\
\hline & 23758 & & 23.5 & $4 \mathrm{f}^{8}\left({ }^{5} \mathrm{G}\right) 5 \mathrm{~d} 6 \mathrm{~s}^{2}{ }^{6} \mathrm{H}$ & 23.1 & $4 \mathrm{f}^{8}\left({ }^{5} \mathrm{G}\right) 5 \mathrm{~d} 6 \mathrm{~s}^{2}{ }^{6} \mathrm{H}$ & 1.291 & & \\
\hline & 24309 & & 20.3 & $4 f^{8}\left({ }^{7} F\right) 5 d^{2} 6 s{ }^{6} I$ & 15.4 & $4 f^{8}\left({ }^{7} \mathrm{~F}\right) 5 \mathrm{~d}^{2} 6 \mathrm{~s}{ }^{6} \mathrm{H}$ & 1.309 & & \\
\hline & 24334 & & 20.4 & $4 f^{8}\left({ }^{7} F\right) 5 d^{2} 6 s^{8} I$ & 12.6 & $4 f^{8}\left({ }^{7} \mathrm{~F}\right) 5 \mathrm{~d}^{2} 6 \mathrm{~s}^{8} \mathrm{H}$ & 1.345 & & \\
\hline & 24384 & & 51.3 & $4 \mathrm{f}^{8}\left({ }^{7} \mathrm{~F}\right) 5 \mathrm{~d}^{3}{ }^{10} \mathrm{I}$ & 24.2 & $4 \mathrm{f}^{8}\left({ }^{7} \mathrm{~F}\right) 5 \mathrm{~d}^{3}{ }^{10} \mathrm{G}$ & 1.433 & & \\
\hline & 25123 & & 52.0 & $4 f^{9}\left({ }^{6} F\right) 6 s 6 p^{8} G$ & 10.9 & $4 \mathrm{f}^{9}\left({ }^{6} \mathrm{H}\right) 6 \mathrm{~s} 6 \mathrm{p}{ }^{6} \mathrm{I}$ & 1.393 & & \\
\hline & 25156 & & 23.4 & $4 \mathrm{f}^{8}\left({ }^{7} \mathrm{~F}\right) 5 \mathrm{~d}^{2} 6 \mathrm{~s}^{8} \mathrm{I}$ & 10.8 & $4 \mathrm{f}^{8}\left({ }^{7} \mathrm{~F}\right) 5 \mathrm{~d}^{2} 6 \mathrm{~s}^{8} \mathrm{H}$ & 1.342 & & \\
\hline & 25598 & & 63.2 & $4 \mathrm{f}^{8}\left({ }^{5} \mathrm{~L}\right) 5 \mathrm{~d} 6 \mathrm{~s}^{2}{ }^{6} \mathrm{~L}$ & 13.8 & $4 \mathrm{f}^{8}\left({ }^{5} \mathrm{~L}\right) 5 \mathrm{~d} 6 \mathrm{~s}^{2}{ }^{6} \mathrm{M}$ & 0.998 & & \\
\hline \multirow[t]{3}{*}{25825.53} & 25803 & 23 & 15.2 & $4 \mathrm{f}^{9}\left({ }^{6} \mathrm{H}\right) 6 \mathrm{~s} 6 \mathrm{p}{ }^{6} \mathrm{I}$ & 9.9 & $4 f^{9}\left({ }^{6} F\right) 6 s 6 p{ }^{8} G$ & 1.324 & 1.246 & -0.078 \\
\hline & 25910 & & 28.0 & $4 \mathrm{f}^{8}\left({ }^{7} \mathrm{~F}\right) 5 \mathrm{~d}^{3}{ }^{10} \mathrm{H}$ & 16.0 & $4 \mathrm{f}^{8}\left({ }^{7} \mathrm{~F}\right) 5 \mathrm{~d}^{3}{ }^{10} \mathrm{G}$ & 1.442 & & \\
\hline & 25971 & & 17.1 & $4 f^{8}\left({ }^{7} F\right) 5 d^{2} 6 s^{8} G$ & 14.0 & $4 \mathrm{f}^{8}\left({ }^{7} \mathrm{~F}\right) 5 \mathrm{~d}^{2} 6 \mathrm{~s}{ }^{6} \mathrm{H}$ & 1.434 & & \\
\hline \multicolumn{10}{|l|}{$J=17 / 2$} \\
\hline 4646.830 & 4656 & -9 & 90.1 & $4 \mathrm{f}^{8}\left({ }^{7} \mathrm{~F}\right) 5 \mathrm{~d} 6 \mathrm{~s}^{2}{ }^{8} \mathrm{H}$ & 2.5 & $4 \mathrm{f}^{8}\left({ }^{7} \mathrm{~F}\right) 5 \mathrm{~d}^{3}{ }^{8} \mathrm{H}$ & 1.407 & 1.406 & -0.001 \\
\hline 8506.710 & 8514 & -8 & 72.9 & $4 \mathrm{f}^{8}\left({ }^{7} \mathrm{~F}\right) 5 \mathrm{~d}^{2} 6 \mathrm{~s}{ }^{10} \mathrm{G}$ & 11.8 & $4 f^{8}\left({ }^{7} F\right) 5 d^{2} 6 s{ }^{10} G$ & 1.517 & 1.530 & 0.013 \\
\hline 11879.20 & 11882 & -3 & 47.0 & $4 \mathrm{f}^{8}\left({ }^{7} \mathrm{~F}\right) 5 \mathrm{~d}^{2} 6 \mathrm{~s}{ }^{10} \mathrm{H}$ & 31.4 & $4 \mathrm{f}^{8}\left({ }^{7} \mathrm{~F}\right) 5 \mathrm{~d}^{2} 6 \mathrm{~s}{ }^{10} \mathrm{I}$ & 1.438 & 1.430 & -0.008 \\
\hline 14016.91 & 14031 & -14 & 39.0 & $4 \mathrm{f}^{8}\left({ }^{7} \mathrm{~F}\right) 5 \mathrm{~d}^{2} 6 \mathrm{~s}{ }^{10} \mathrm{I}$ & 30.2 & $4 \mathrm{f}^{8}\left({ }^{7} \mathrm{~F}\right) 5 \mathrm{~d}^{2} 6 \mathrm{~s}{ }^{10} \mathrm{H}$ & 1.415 & & \\
\hline 14718.11 & 14739 & -21 & 47.4 & $4 \mathrm{f}^{8}\left({ }^{7} \mathrm{~F}\right) 5 \mathrm{~d}^{2} 6 \mathrm{~s}^{8} \mathrm{H}$ & 14.7 & $4 f^{8}\left({ }^{7} \mathrm{~F}\right) 5 \mathrm{~d}^{2} 6 \mathrm{~s}{ }^{10} \mathrm{I}$ & 1.395 & 1.400 & 0.005 \\
\hline \multirow[t]{3}{*}{15189.26} & 15166 & 24 & 45.2 & $4 \mathrm{f}^{9}\left({ }^{6} \mathrm{H}\right) 6 \mathrm{~s} 6 \mathrm{p}{ }^{8} \mathrm{H}$ & 13.6 & $4 \mathrm{f}^{9}\left({ }^{6} \mathrm{H}\right) 6 \mathrm{~s} 6 \mathrm{p}{ }^{8} \mathrm{I}$ & 1.367 & 1.409 & 0.042 \\
\hline & 15699 & & 60.4 & $4 \mathrm{f}^{8}\left({ }^{7} \mathrm{~F}\right) 5 \mathrm{~d}^{2} 6 \mathrm{~s}{ }^{10} \mathrm{G}$ & 15.8 & $4 \mathrm{f}^{8}\left({ }^{7} \mathrm{~F}\right) 5 \mathrm{~d}^{2} 6 \mathrm{~s}{ }^{10} \mathrm{G}$ & 1.494 & & \\
\hline & 16766 & & 31.8 & $4 \mathrm{f}^{8}\left({ }^{7} \mathrm{~F}\right) 5 \mathrm{~d}^{2} 6 \mathrm{~s}^{8} \mathrm{I}$ & 19.7 & $4 \mathrm{f}^{8}\left({ }^{7} \mathrm{~F}\right) 5 \mathrm{~d}^{2} 6 \mathrm{~s}^{8} \mathrm{H}$ & 1.361 & & \\
\hline
\end{tabular}


Table 1. Continued.

\begin{tabular}{|c|c|c|c|c|c|c|c|c|c|}
\hline$E_{\exp }$ & $E_{\text {calc }}$ & $\Delta E$ & $\%$ & Main comp. & $\%$ & Sec. comp. & $g_{J \text { calc }}$ & $g_{J \exp }$ & $\Delta g_{J}$ \\
\hline \multirow[t]{9}{*}{17249.59} & 17260 & -11 & 44.7 & $4 \mathrm{f}^{9}\left({ }^{6} \mathrm{H}\right) 6 \mathrm{~s} 6 \mathrm{p}{ }^{6} \mathrm{I}$ & 30.0 & $4 \mathrm{f}^{9}\left({ }^{6} \mathrm{H}\right) 6 \mathrm{~s} 6 \mathrm{p}^{8} \mathrm{H}$ & 1.329 & & \\
\hline & 18798 & & 27.9 & $4 \mathrm{f}^{8}\left({ }^{7} \mathrm{~F}\right) 5 \mathrm{~d}^{2} 6 \mathrm{~s}{ }^{6} \mathrm{I}$ & 24.1 & $4 f^{8}\left({ }^{7} \mathrm{~F}\right) 5 \mathrm{~d}^{2} 6 \mathrm{~s}^{8} \mathrm{H}$ & 1.355 & & \\
\hline & 19491 & & 24.4 & $4 \mathrm{f}^{8}\left({ }^{7} \mathrm{~F}\right) 5 \mathrm{~d}^{2} 6 \mathrm{~s}^{8} \mathrm{H}$ & 21.9 & $4 f^{8}\left({ }^{7} F\right) 5 d^{2} 6 s{ }^{6} I$ & 1.353 & & \\
\hline & 20287 & & 18.3 & $4 \mathrm{f}^{8}\left({ }^{7} \mathrm{~F}\right) 5 \mathrm{~d}^{2} 6 \mathrm{~s}^{8} \mathrm{I}$ & 17.0 & $4 f^{8}\left({ }^{7} F\right) 5 d^{2} 6 s^{8} I$ & 1.356 & & \\
\hline & 20702 & & 51.3 & $4 \mathrm{f}^{9}\left({ }^{6} \mathrm{H}\right) 6 \mathrm{~s} 6 \mathrm{p}{ }^{8} \mathrm{I}$ & 11.2 & $4 \mathrm{f}^{9}\left({ }^{6} \mathrm{H}\right) 6 \mathrm{~s} 6 \mathrm{p}{ }^{6} \mathrm{I}$ & 1.329 & & \\
\hline & 21413 & & 35.5 & $4 \mathrm{f}^{9}\left({ }^{6} \mathrm{H}\right) 6 \mathrm{~s} 6 \mathrm{p}{ }^{6} \mathrm{I}$ & 12.6 & $4 f^{8}\left({ }^{7} F\right) 5 d^{2} 6 s{ }^{6} I$ & 1.300 & & \\
\hline & 21854 & & 45.9 & $4 \mathrm{f}^{8}\left({ }^{7} \mathrm{~F}\right) 5 \mathrm{~d}^{3}{ }^{10} \mathrm{H}$ & 26.2 & $4 \mathrm{f}^{8}\left({ }^{7} \mathrm{~F}\right) 5 \mathrm{~d}^{3}{ }^{10} \mathrm{I}$ & 1.447 & & \\
\hline & 22233 & & 31.3 & $4 \mathrm{f}^{8}\left({ }^{7} \mathrm{~F}\right) 5 \mathrm{~d}^{2} 6 \mathrm{~s}^{8} \mathrm{H}$ & 10.0 & $4 f^{8}\left({ }^{7} F\right) 5 d^{2} 6 s^{8} I$ & 1.371 & & \\
\hline & 22875 & & 36.7 & $4 \mathrm{f}^{8}\left({ }^{7} \mathrm{~F}\right) 5 \mathrm{~d}^{2} 6 \mathrm{~s}^{8} \mathrm{H}$ & 18.7 & $4 f^{8}\left({ }^{7} F\right) 5 d^{2} 6 s^{8} I$ & 1.373 & & \\
\hline \multirow[t]{4}{*}{23107.25} & 23146 & -39 & 34.0 & $4 \mathrm{f}^{8}\left({ }^{7} \mathrm{~F}\right) 5 \mathrm{~d}^{2} 6 \mathrm{~s}{ }^{6} \mathrm{I}$ & 11.7 & $4 f^{8}\left({ }^{7} F\right) 5 d^{2} 6 s^{8} I$ & 1.307 & 1.289 & -0.018 \\
\hline & 23681 & & 45.9 & $4 \mathrm{f}^{8}\left({ }^{7} \mathrm{~F}\right) 5 \mathrm{~d}^{3}{ }^{10} \mathrm{I}$ & 31.8 & $4 f^{8}\left({ }^{7} F\right) 5 d^{3}{ }^{10} G$ & 1.453 & & \\
\hline & 24512 & & 33.6 & $4 \mathrm{f}^{8}\left({ }^{7} \mathrm{~F}\right) 5 \mathrm{~d}^{2} 6 \mathrm{~s}^{8} \mathrm{I}$ & 14.8 & $4 \mathrm{f}^{8}\left({ }^{7} \mathrm{~F}\right) 5 \mathrm{~d}^{2} 6 \mathrm{~s}^{8} \mathrm{H}$ & 1.347 & & \\
\hline & 24978 & & 53.5 & $4 f^{8}\left({ }^{5} \mathrm{~L}\right) 5 \mathrm{~d} 6 \mathrm{~s}^{2}{ }^{6} \mathrm{~L}$ & 19.6 & $4 \mathrm{f}^{8}\left({ }^{5} \mathrm{~L}\right) 5 \mathrm{~d} 6 \mathrm{~s}^{2}{ }^{6} \mathrm{~K}$ & 1.127 & & \\
\hline \multicolumn{10}{|l|}{$J=19 / 2$} \\
\hline 11331.14 & 11313 & 18 & 65.8 & $4 \mathrm{f}^{8}\left({ }^{7} \mathrm{~F}\right) 5 \mathrm{~d}^{2} 6 \mathrm{~s}{ }^{10} \mathrm{H}$ & 28.5 & $4 \mathrm{f}^{8}\left({ }^{7} \mathrm{~F}\right) 5 \mathrm{~d}^{2} 6 \mathrm{~s}{ }^{10} \mathrm{I}$ & 1.451 & 1.460 & 0.009 \\
\hline \multirow[t]{6}{*}{13398.40} & 13395 & 3 & 56.7 & $4 \mathrm{f}^{8}\left({ }^{7} \mathrm{~F}\right) 5 \mathrm{~d}^{2} 6 \mathrm{~s}{ }^{10} \mathrm{I}$ & 27.5 & $4 \mathrm{f}^{8}\left({ }^{7} \mathrm{~F}\right) 5 \mathrm{~d}^{2} 6 \mathrm{~s}{ }^{10} \mathrm{H}$ & 1.422 & & \\
\hline & 15870 & & 68.5 & $4 \mathrm{f}^{8}\left({ }^{7} \mathrm{~F}\right) 5 \mathrm{~d}^{2} 6 \mathrm{~s}^{8} \mathrm{I}$ & 11.8 & $4 f^{8}\left({ }^{7} F\right) 5 d^{2} 6 s^{8} I$ & 1.372 & & \\
\hline & 16792 & & 86.6 & $4 \mathrm{f}^{9}\left({ }^{6} \mathrm{H}\right) 6 \mathrm{~s} 6 \mathrm{p}{ }^{8} \mathrm{I}$ & 7.1 & $4 \mathrm{f}^{9}\left({ }^{4} \mathrm{I}\right) 6 \mathrm{~s} 6 \mathrm{p}{ }^{6} \mathrm{~K}$ & 1.358 & & \\
\hline & 20295 & & 55.8 & $4 \mathrm{f}^{8}\left({ }^{7} \mathrm{~F}\right) 5 \mathrm{~d}^{2} 6 \mathrm{~s}^{8} \mathrm{I}$ & 29.6 & $4 \mathrm{f}^{8}\left({ }^{7} \mathrm{~F}\right) 5 \mathrm{~d}^{2} 6 \mathrm{~s}^{8} \mathrm{I}$ & 1.363 & & \\
\hline & 21715 & & 48.5 & $4 \mathrm{f}^{8}\left({ }^{7} \mathrm{~F}\right) 5 \mathrm{~d}^{3}{ }^{10} \mathrm{I}$ & 44.5 & $4 \mathrm{f}^{8}\left({ }^{7} \mathrm{~F}\right) 5 \mathrm{~d}^{3}{ }^{10} \mathrm{H}$ & 1.436 & & \\
\hline & 22984 & & 49.6 & $4 \mathrm{f}^{8}\left({ }^{7} \mathrm{~F}\right) 5 \mathrm{~d}^{2} 6 \mathrm{~s}^{8} \mathrm{I}$ & 20.9 & $4 f^{8}\left({ }^{7} F\right) 5 d^{2} 6 s^{8} I$ & 1.363 & & \\
\hline \multicolumn{10}{|l|}{$J=21 / 2$} \\
\hline \multirow[t]{4}{*}{12283.30} & 12248 & 36 & 95.2 & $4 \mathrm{f}^{8}\left({ }^{7} \mathrm{~F}\right) 5 \mathrm{~d}^{2} 6 \mathrm{~s}{ }^{10} \mathrm{I}$ & 2.0 & $4 \mathrm{f}^{8}\left({ }^{5} \mathrm{G}\right) 5 \mathrm{~d}^{2} 6 \mathrm{~s}^{8} \mathrm{~K}$ & 1.425 & & \\
\hline & 21743 & & 93.3 & $4 \mathrm{f}^{8}\left({ }^{7} \mathrm{~F}\right) 5 \mathrm{~d}^{3}{ }^{10} \mathrm{I}$ & 2.6 & $4 \mathrm{f}^{8}\left({ }^{5} \mathrm{G}\right) 5 \mathrm{~d}^{3}{ }^{8} \mathrm{~K}$ & 1.423 & & \\
\hline & 23610 & & 69.4 & $4 \mathrm{f}^{8}\left({ }^{5} \mathrm{~L}\right) 5 \mathrm{~d} 6 \mathrm{~s}^{2}{ }^{6} \mathrm{~L}$ & 13.0 & $4 \mathrm{f}^{8}\left({ }^{5} \mathrm{~L}\right) 5 \mathrm{~d} 6 \mathrm{~s}^{2}{ }^{6} \mathrm{M}$ & 1.215 & & \\
\hline & 24720 & & 88.1 & $4 \mathrm{f}^{8}\left({ }^{7} \mathrm{~F}\right) 5 \mathrm{~d}^{2} 6 \mathrm{~s}{ }^{8} \mathrm{~K}$ & 4.2 & $4 \mathrm{f}^{8}\left({ }^{7} \mathrm{~F}\right) 5 \mathrm{~d}^{3}{ }^{8} \mathrm{~K}$ & 1.330 & & \\
\hline \multicolumn{10}{|l|}{$J=23 / 2$} \\
\hline & 24760 & & 50.1 & $4 \mathrm{f}^{8}\left({ }^{5} \mathrm{~L}\right) 5 \mathrm{~d} 6 \mathrm{~s}^{2}{ }^{6} \mathrm{M}$ & 21.8 & $4 \mathrm{f}^{8}\left({ }^{5} \mathrm{~L}\right) 5 \mathrm{~d} 6 \mathrm{~s}^{2}{ }^{6} \mathrm{~N}$ & 1.179 & & \\
\hline \multicolumn{10}{|l|}{$J=25 / 2$} \\
\hline & 26160 & & 88.0 & $4 \mathrm{f}^{8}\left({ }^{5} \mathrm{~L}\right) 5 \mathrm{~d} 6 \mathrm{~s}^{2}{ }^{6} \mathrm{~N}$ & 4.1 & $4 \mathrm{f}^{8}\left({ }^{3} \mathrm{M}\right) 5 \mathrm{~d} 6 \mathrm{~s}^{2}{ }^{4} \mathrm{O}$ & 1.194 & & \\
\hline \multicolumn{10}{|l|}{$J=27 / 2$} \\
\hline & 33634 & & 80.3 & $4 \mathrm{f}^{8}\left({ }^{5} \mathrm{~L}\right) 5 \mathrm{~d}^{2} 6 \mathrm{~s}^{8} \mathrm{~N}$ & 11.8 & $4 \mathrm{f}^{8}\left({ }^{5} \mathrm{~L}\right) 5 \mathrm{~d}^{2} 6 \mathrm{~s}{ }^{8} \mathrm{O}$ & 1.247 & & \\
\hline \multicolumn{10}{|l|}{$J=29 / 2$} \\
\hline & 35024 & & 92.4 & $4 f^{8}\left({ }^{5} L\right) 5 d^{2} 6 s^{8} O$ & 4.0 & $4 \mathrm{f}^{8}\left({ }^{3} \mathrm{M}\right) 5 \mathrm{~d}^{2} 6 \mathrm{~s}{ }^{6} \mathrm{Q}$ & 1.236 & & \\
\hline \multicolumn{10}{|l|}{$J=31 / 2$} \\
\hline & 48950 & & 50.9 & $4 \mathrm{f}^{8}\left({ }^{3} \mathrm{O}\right) 5 \mathrm{~d}^{2} 6 \mathrm{~s}^{6} \mathrm{~T}$ & 36.3 & $4 \mathrm{f}^{8}\left({ }^{3} \mathrm{O}\right) 5 \mathrm{~d}^{2} 6 \mathrm{~s}^{6} \mathrm{R}$ & 1.125 & & \\
\hline
\end{tabular}

Open Access This is an open access article distributed under the terms of the Creative Commons Attribution License (http://creativecommons.org/licenses/by/4.0), which permits unrestricted use, distribution, and reproduction in any medium, provided the original work is properly cited. 


\section{References}

1. M. Elantkowska, J. Ruczkowski, J. Dembczyński, Eur. Phys. J. Plus 130, 14 (2015).

2. M. Elantkowska, J. Ruczkowski, J. Dembczyński, Eur. Phys. J. Plus 130, 15 (2015).

3. M. Elantkowska, J. Ruczkowski, J. Dembczyński, Eur. Phys. J. Plus 130, 83 (2015).

4. M. Elantkowska, J. Ruczkowski, J. Dembczyński, Eur. Phys. J. Plus 130, 170 (2015).

5. M. Elantkowska, J. Ruczkowski, J. Dembczyński, Eur. Phys. J. Plus 131, 47 (2016).

6. M. Elantkowska, J. Ruczkowski, J. Dembczyński, Eur. Phys. J. Plus 131, 429 (2016).

7. J. Dembczyński, M. Elantkowska, K. Bekk, H. Rebel, M. Wilson, Z. Phys. D 13, 181 (1989).

8. M. Elantkowska, A. Bernard, J. Dembczyński, J. Ruczkowski, Z. Phys. D 27, 103 (1993).

9. E. Stachowska, M. Elantkowska, J. Ruczkowski, J. Dembczyński, Phys. Scr. 65, 237 (2002).

10. J. Ruczkowski, E. Stachowska, M. Elantkowska, G.H. Guthöhrlein, J. Dembczyński, Phys. Scr. 68, 133 (2003).

11. B. Furmann, D. Stefanska, A. Krzykowski, Spectrochim. Acta Part B 111, 38 (2015).

12. B. Furmann, D. Stefanska, A. Krzykowski, J. Phys. B 49, 025001 (2016).

13. D. Stefanska, M. Elantkowska, J. Ruczkowski, B. Furmann, J. Quantum Spectrosc. Radiat. Transf. 189, 441 (2017).

14. Z.G. Zhang, S. Svanberg, P. Palmeri, P. Quinet, E. Biemont, Mon. Not. R. Astron. Soc. 334, 1 (2002).

15. R.D. Cowan, The Theory of Atomic Structure and Spectra (Berkeley University of California Press, Berkeley, 1981).

16. R.D. Cowan, Robert D. Cowan's Atomic Structure Code, available on-line at the following link: https://www.tcd.ie/Physics/people/Cormac.McGuinness/Cowan/.

17. P. Quinet, E. Biémont, Mon. Not. R. Astron. Soc. 340, 463 (2003).

18. J.-F. Wyart, W.-Ü.L. Tchang-Brillet, S.S. Churilov, A.N. Ryabtsev, Astron. Astrophys. 483, 339 (2008).

19. M. Macy et al., available on-line: https://docs.microsoft.com/pl-pl/azure/batch/batch-mpi.

20. A. Kramida, Yu. Ralchenko, J. Reader, NIST ASD Team, NIST Atomic Spectra Database (ver. 5.3), available on-line: http://physics.nist.gov/asd (National Institute of Standards and Technology, Gaithersburg, MD, 2016).

21. W.C. Martin, R. Zalubas, L. Hagan, Atomic energy levels - The rare-earth elements, in Nat. Stand. Ref. Data Ser., NSRDSNBS 60, Nat. Bur. Stand., U.S. (1978). 